\title{
Supporting Information: Finding Druggable Sites in Proteins using TACTICS
}

\author{
Daniel J. Evans ${ }^{1 \pi}$, Remy A. Yovanno1ึ, Sanim Rahman¹ ${ }^{1 \pi}$ David W. Cao ${ }^{1}$, Morgan Q. Beckett ${ }^{2}$, \\ Milan H. Patel ${ }^{1}$, Afif F. Bandak¹, Albert Y. Lau ${ }^{*}$
}

${ }^{1}$ Department of Biophysics and Biophysical Chemistry

Johns Hopkins University School of Medicine

Baltimore, MD 21205, USA

${ }^{2}$ Department of Biochemistry and Molecular Biology

Johns Hopkins Bloomberg School of Public Health

Baltimore, MD 21205, USA

TThese authors contributed equally.

${ }^{*}$ To whom correspondence should be addressed:

alau@jhmi.edu 
A

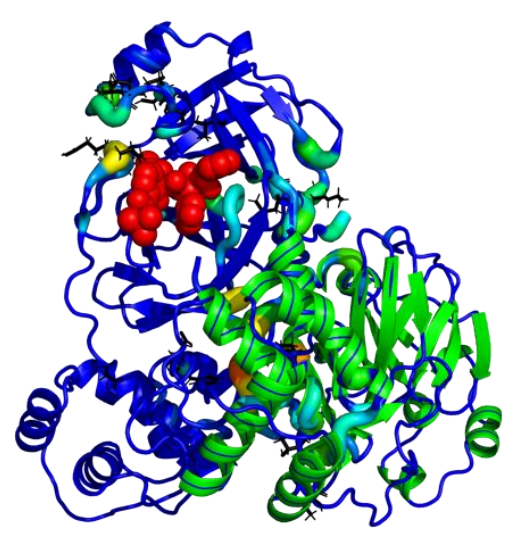

C

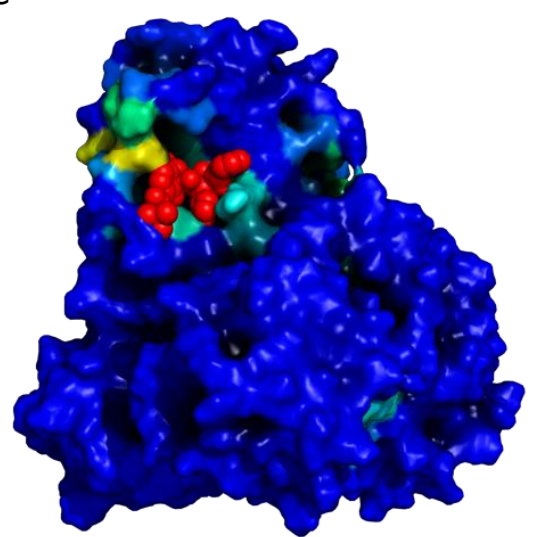

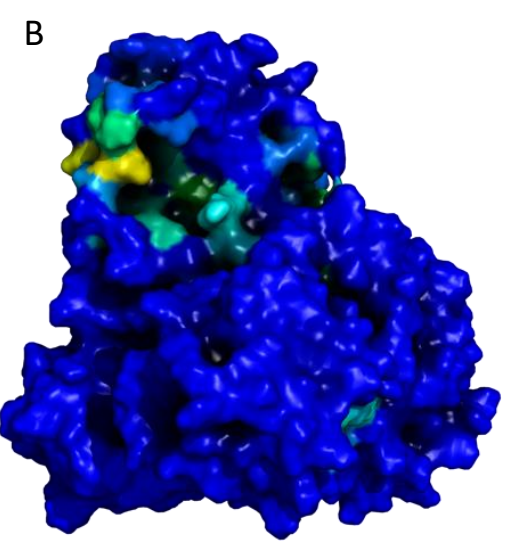

D

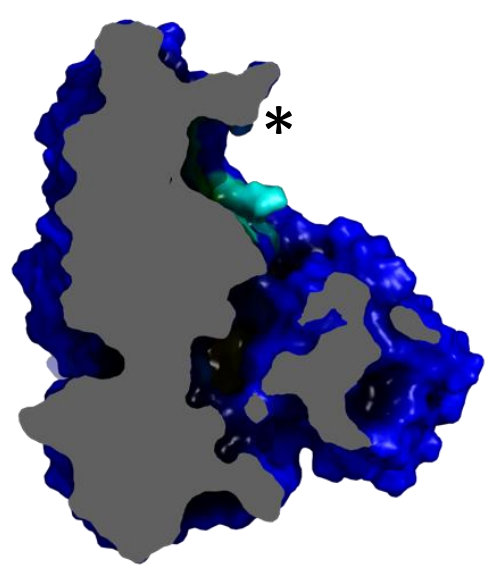

Figure S1. Additional images of TACTICS output showing the SARS-CoV-2 main protease active site. A. The same as Figure (2B), with the protomers colored to show tertiary structure. B. Surface view of the pocket, with residues colored by TACTICS fragment docking scores. C. The same as (B), with an active-site inhibitor called $13 \mathrm{~b}$ added from PDB ID 6Y2F. D. Rotated view of the protein. The asterisk indicates the active site location. 
A

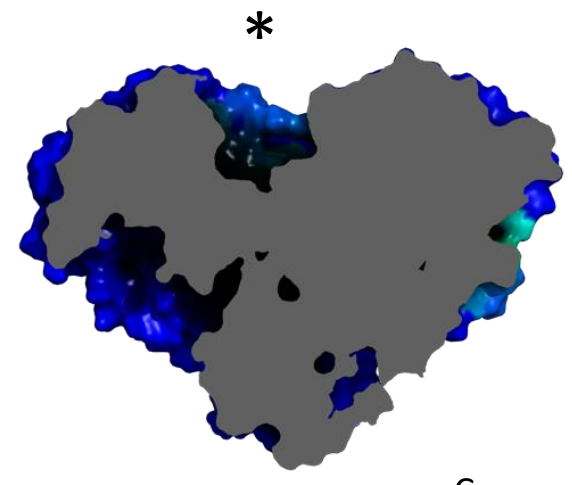

B

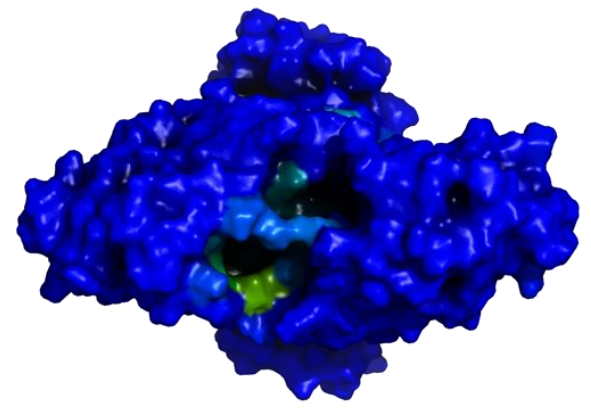

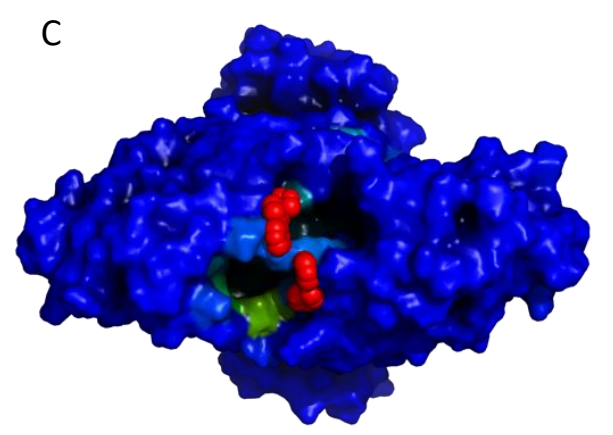

Figure S2. Additional images of TACTICS output showing a known allosteric site in the SARSCoV-2 main protease. A. Slice representation of the protein. The pocket location is indicated by the asterisk. B. Surface view of the top of the pocket, with residues colored by TACTICS fragment docking scores. C. The same as (B), with an inhibitor called $\times 0425$ or Z1401276297 added from PDB ID 5RGJ.
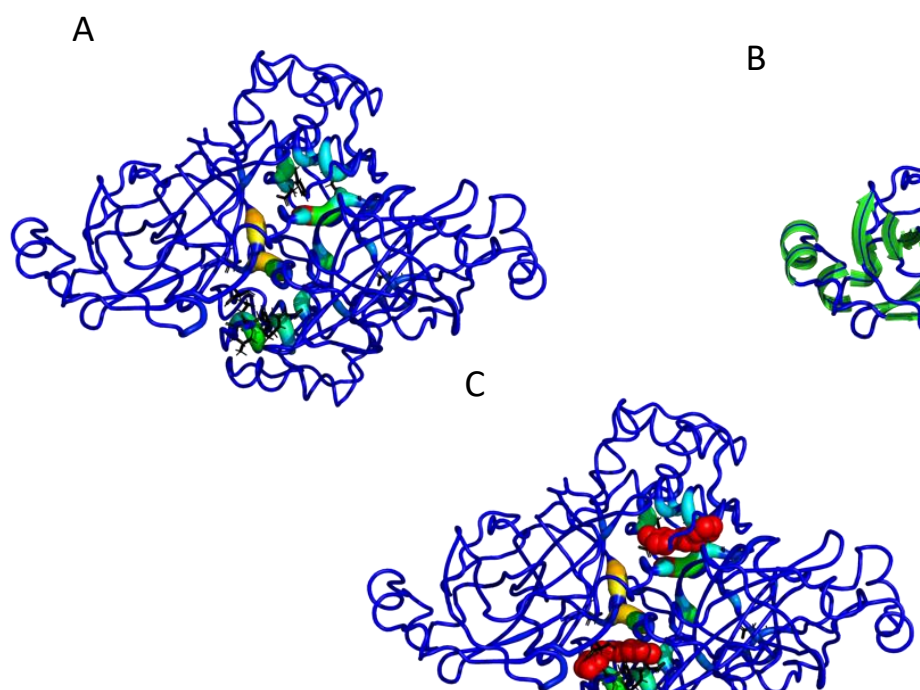

B

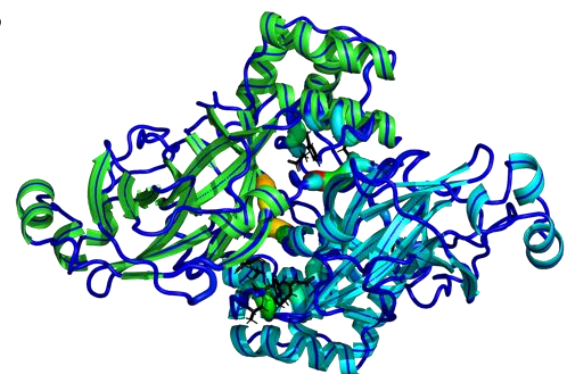

Figure S3. TACTICS finds a known binding

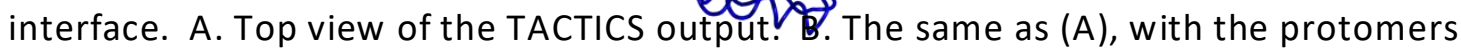
colored to show the tertiary structure. $C$. The same as $(A)$, with an inhibitor called $\times 1187$ or Z2643472210 added from PDB ID 5RFA. 

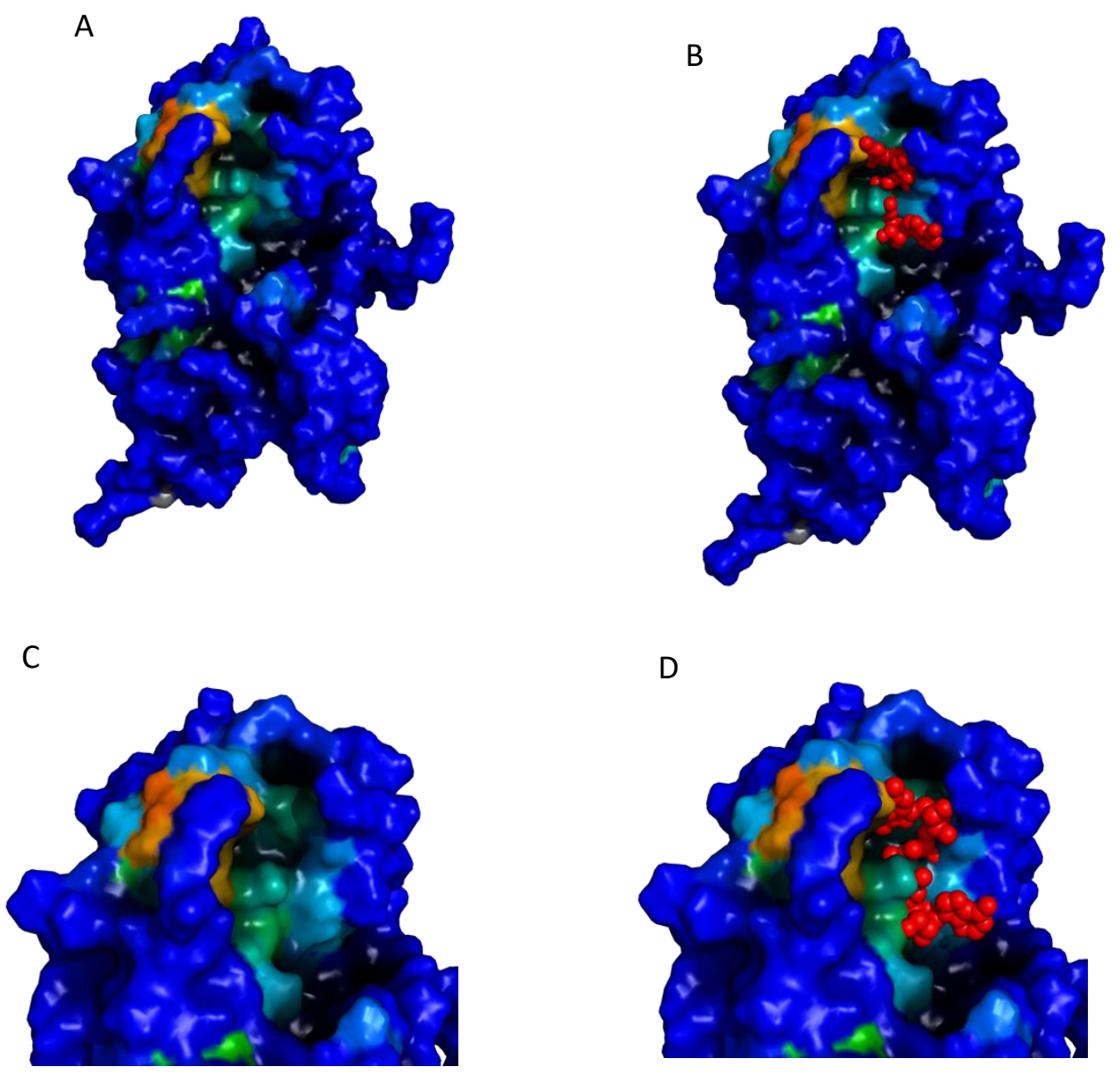

$\mathrm{F}$

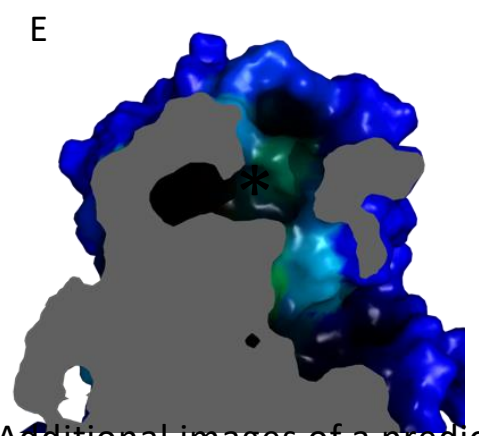

Figure S4. Adeditional images of a predicted site connected to the NTS se RNA binding site. A. Surface view of the structure shown in figure 3C. B. Surface view with $m^{\prime} G p p p A$ added from PDB structure 6WVN. C,D. Slightly rotated close-up of binding site. E. Slice view of site. The asterisk notes the area where the binding site is. Notice the cavity on the left; its opening is controlled by L27, S202, and nearby residues. F. An alignment of two frames from the MD data. L27 and S202 are shown in sticks. In the green frame, the residues are close to each other, blocking part of the cavity. In the blue frame, the residues are farther apart, allowing for access. Some parts of the protein were removed for image clarity. 


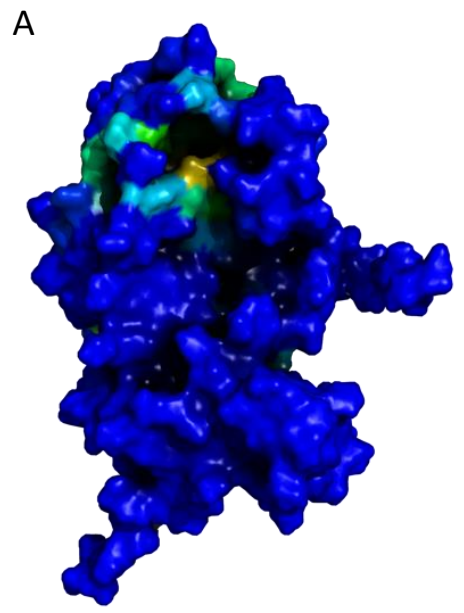

B
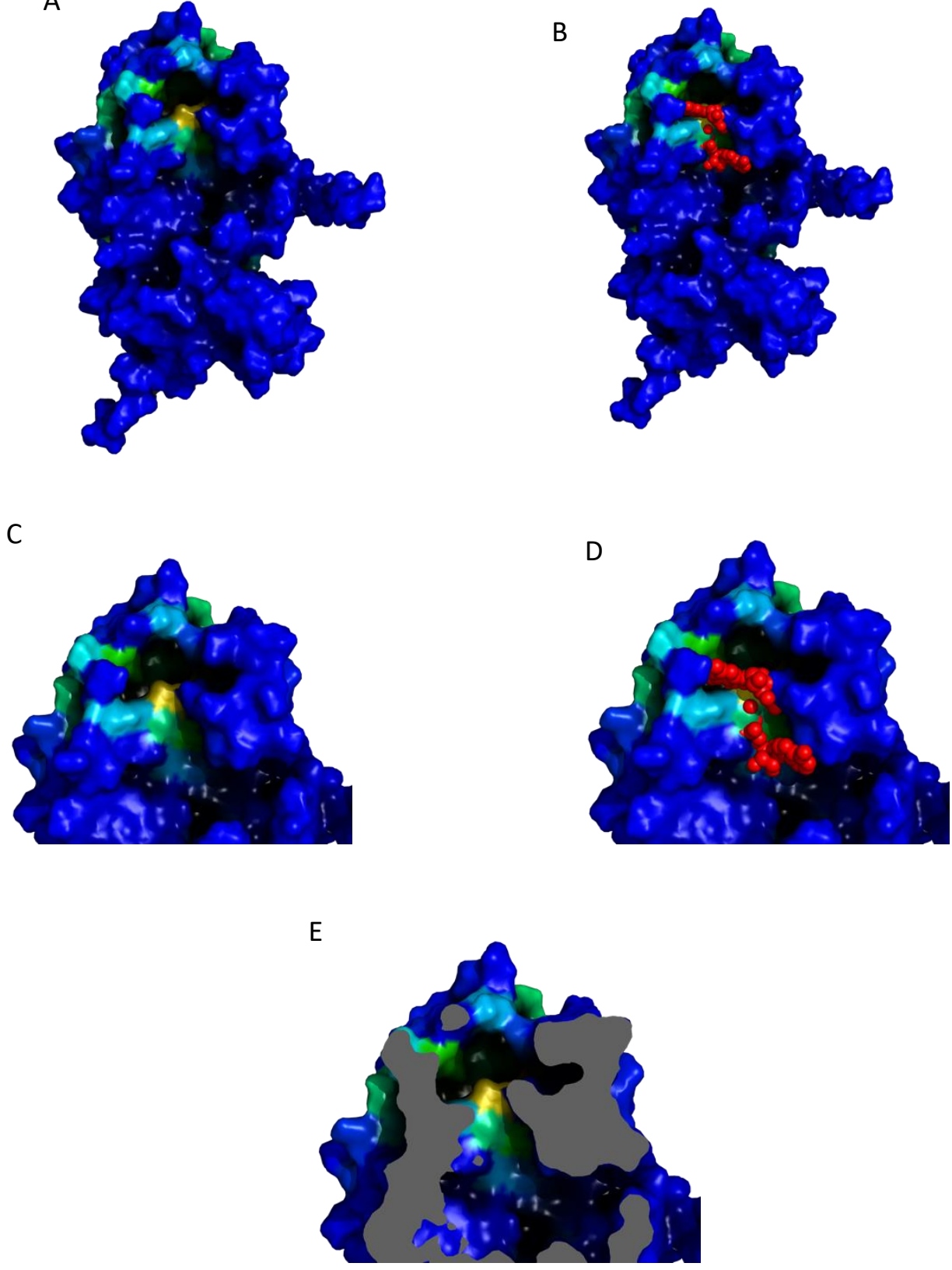

Figure S5. Additional images of a predicted site connected to the MTase RNA binding site. This site is different from the one seen in Figure S4. A. Surface view of the structure shown in figure $3 \mathrm{E}$. B. The same as (A), with $\mathrm{m}^{7} \mathrm{GpppA}$ added from PDB structure 6WVN. C, D. Slightly rotated close-up of binding site. E. Slice view of site. Notice the cavity at the top right. 
A

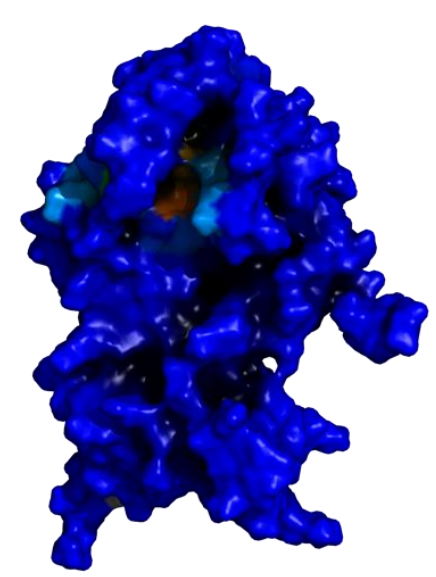

B

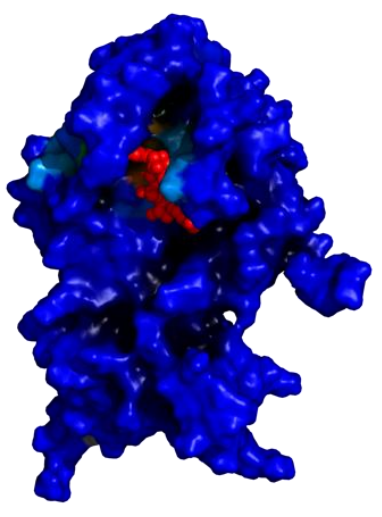

Figure S6. Additional images of the SAM site as identified by TACTICS. A. Surface representation of Figure 4A. B. Surface representation of Figure 4B. 
A
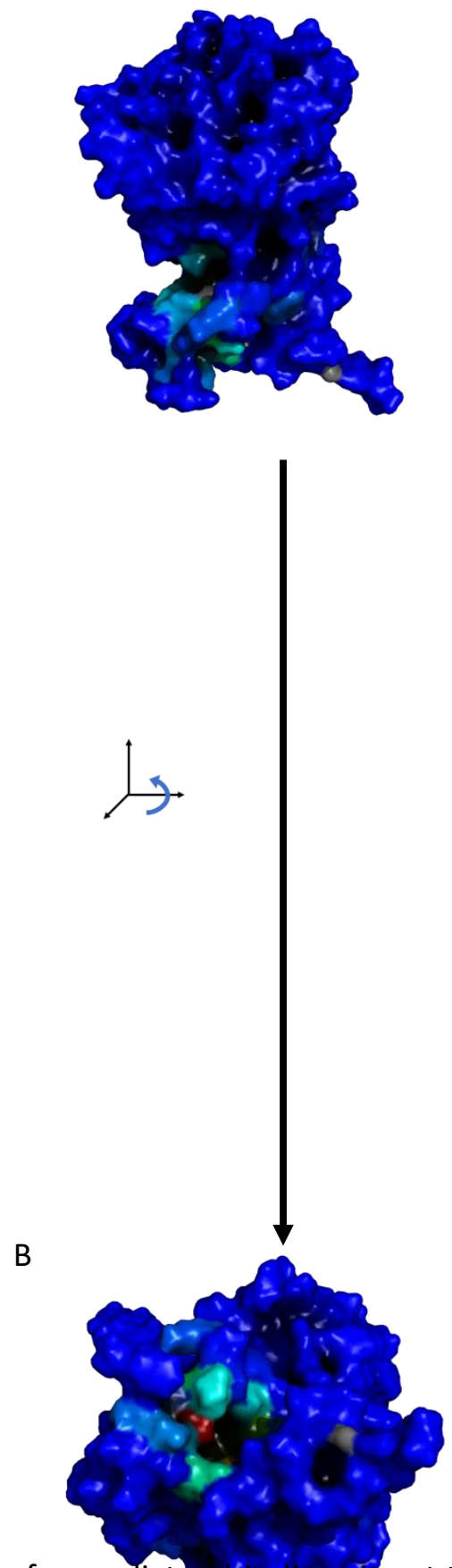

C

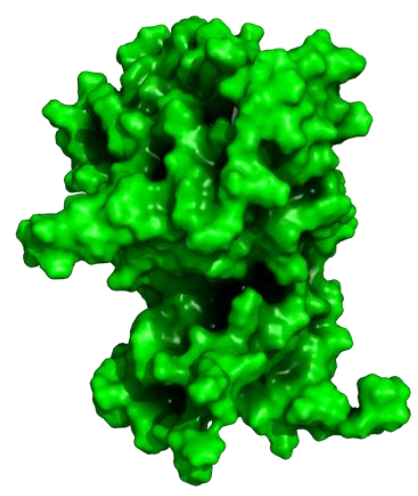

D
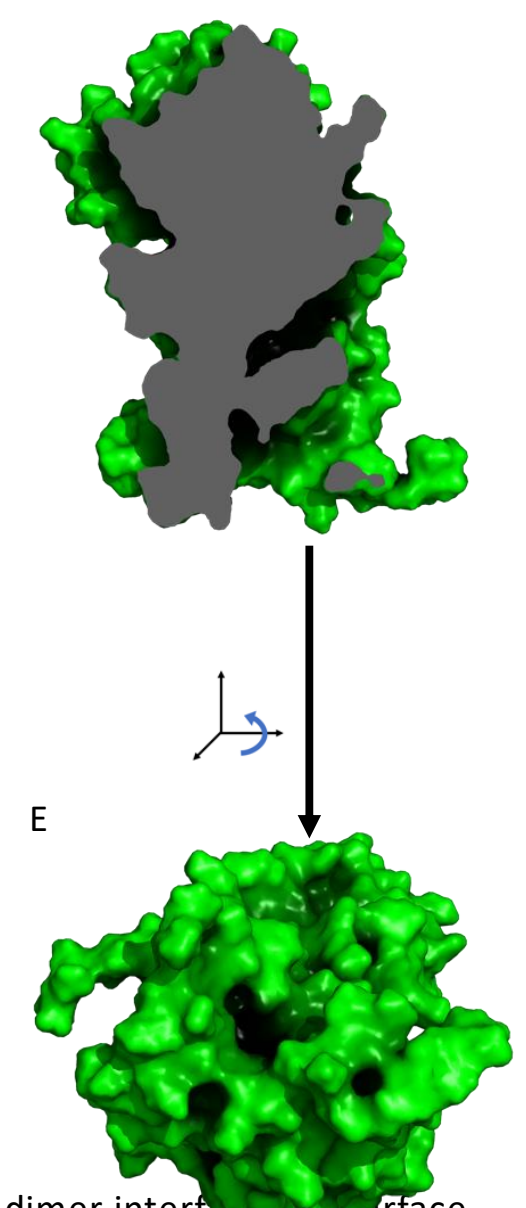

Figure S7. Additional images of a predicte site at the dimer interf surface view of MTase. The predicted binding site is at the bottom left; it is colored bYTACTICS. B. Rotated surface view showing the binding site. C. Surface view of the first frame of the MD data. D. Slice representation of the first frame of the MD data. Note that the tunnel is not fully present. E. Rotated view of the first frame. 
A

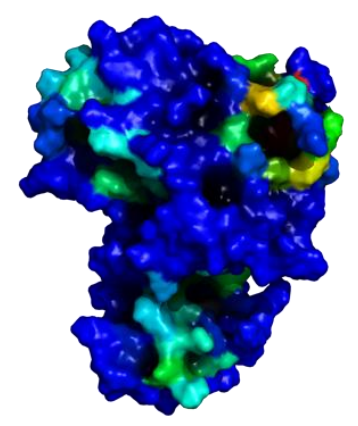

B
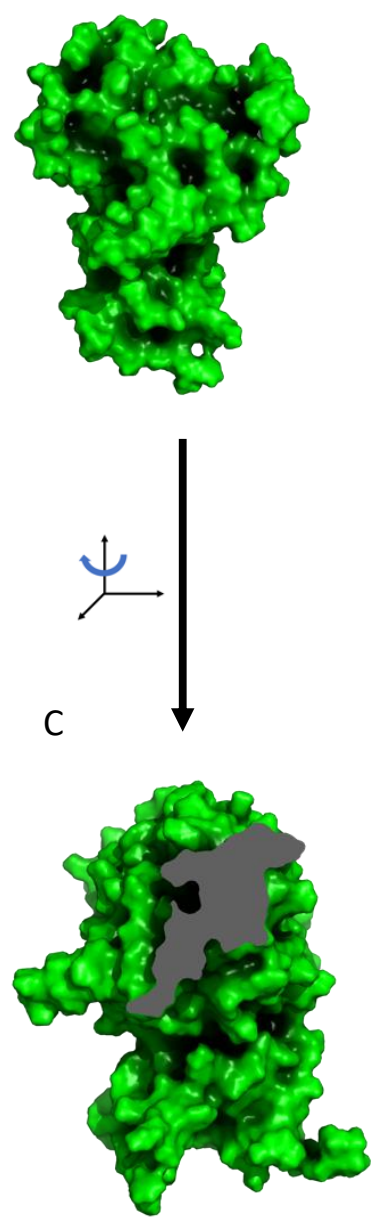

Figure S8. Additional images of a predicted binding site on the methyltransferase. A. Surface view of MTase. The predicted site of interest here is on the right. The RNA site is on the left. B. Surface view of the first frame of the MD data. C. Slice representation of the first frame of the MD data. 
A

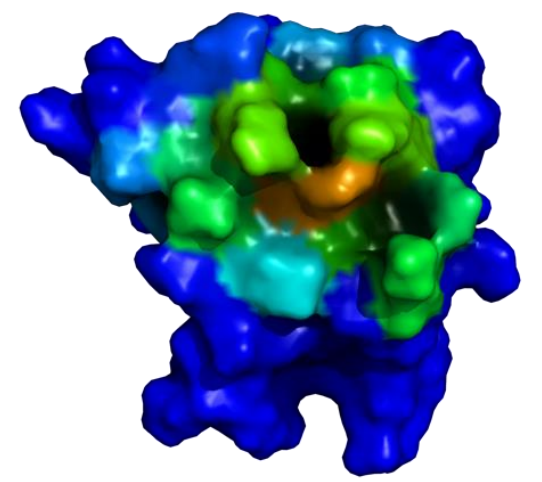

B

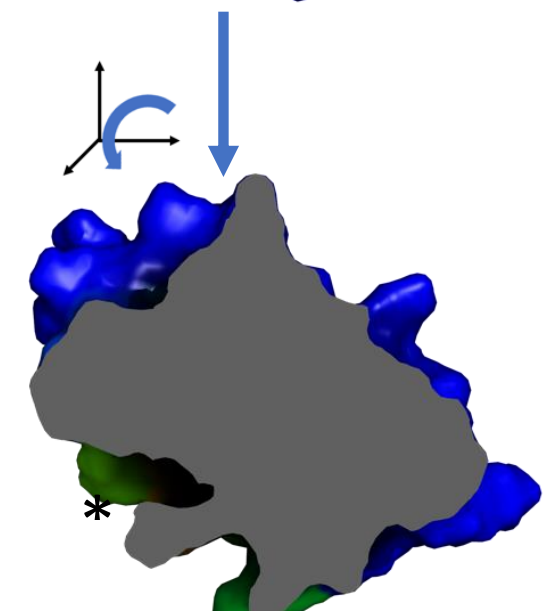

$C$

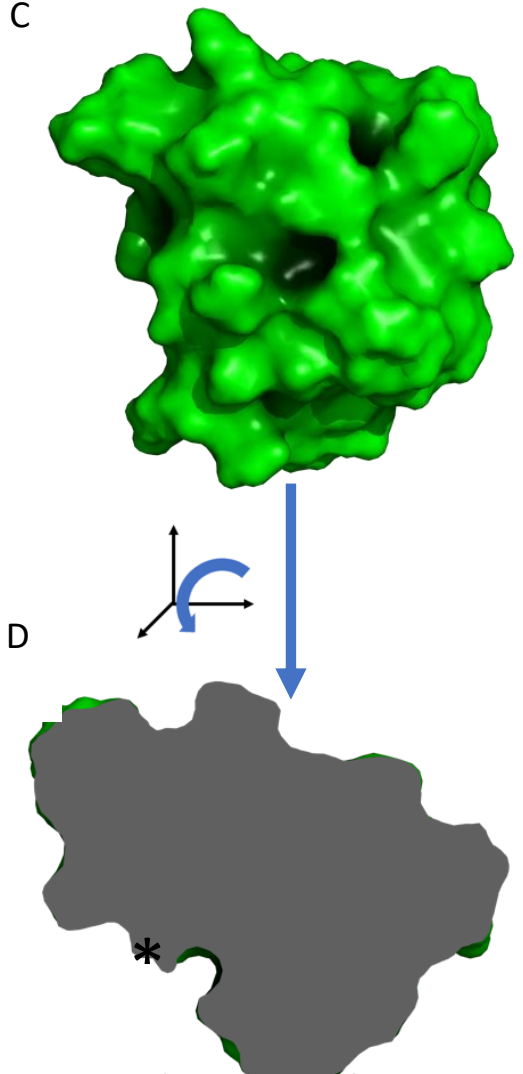

Figure S9. Additionatimages of the apo-ArCP predicted site shown in Figure 5 A-B. A. Surface representation of Figure 5A. B. Rotated slice representation of this conformation. The asterisk marks the predicted site location. C. Surface view of the first frame of the MD trajectory, aligned with (A). D. Slice view of the first frame of the MD trajectory. The asterisk marks the predicted site location. 

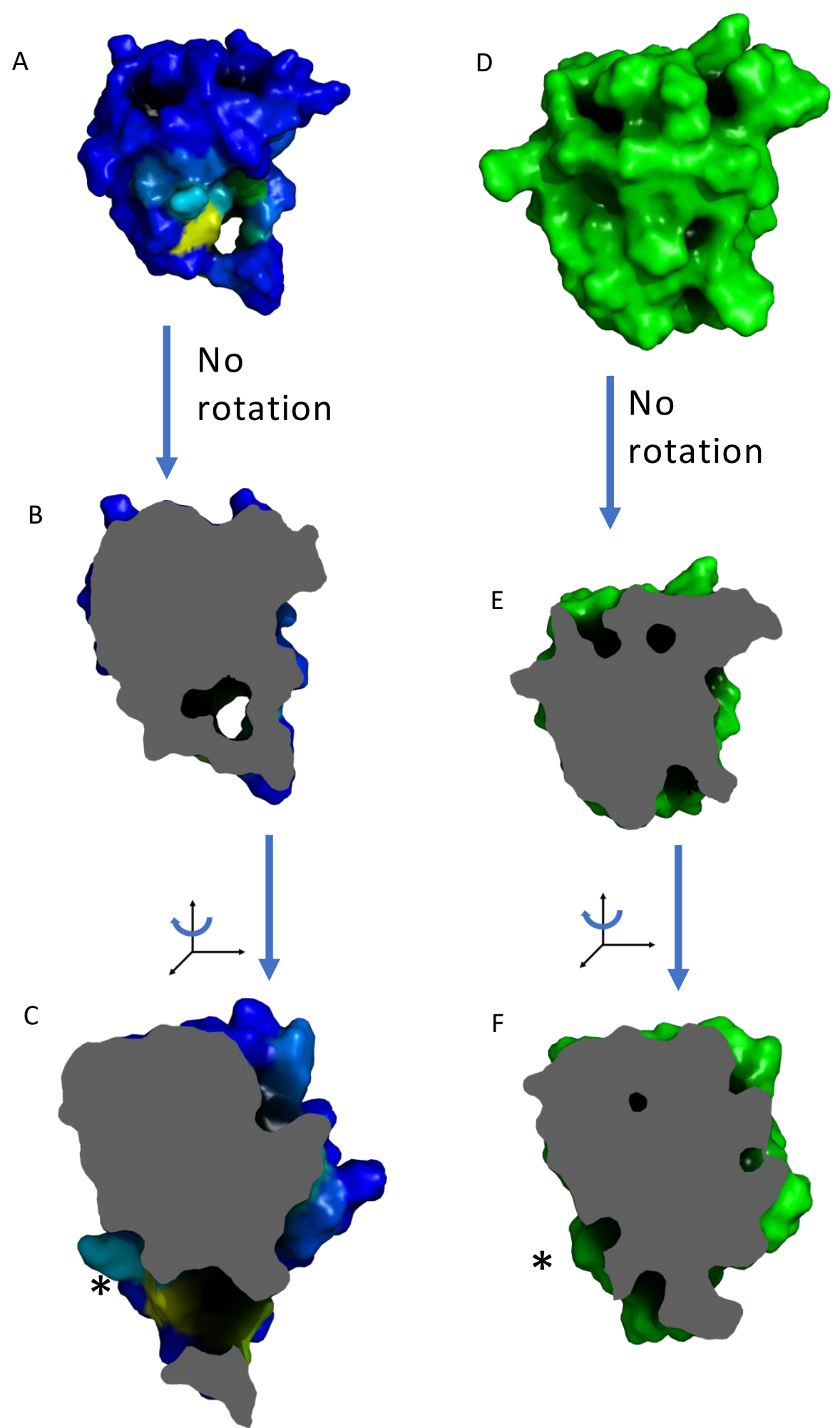

Figure S10. Additional images of the apo-ArCP predicted site shown in Figure 5 C-D. A. Surface representation of Figure $5 C$. B, C. Slice views of this frame. The asterisk indicates the predicted site's location. D. Surface view of the first frame of the MD trajectory, aligned with (A). E, F. Slice views of the first frame. The asterisk indicates the predicted site's location. 

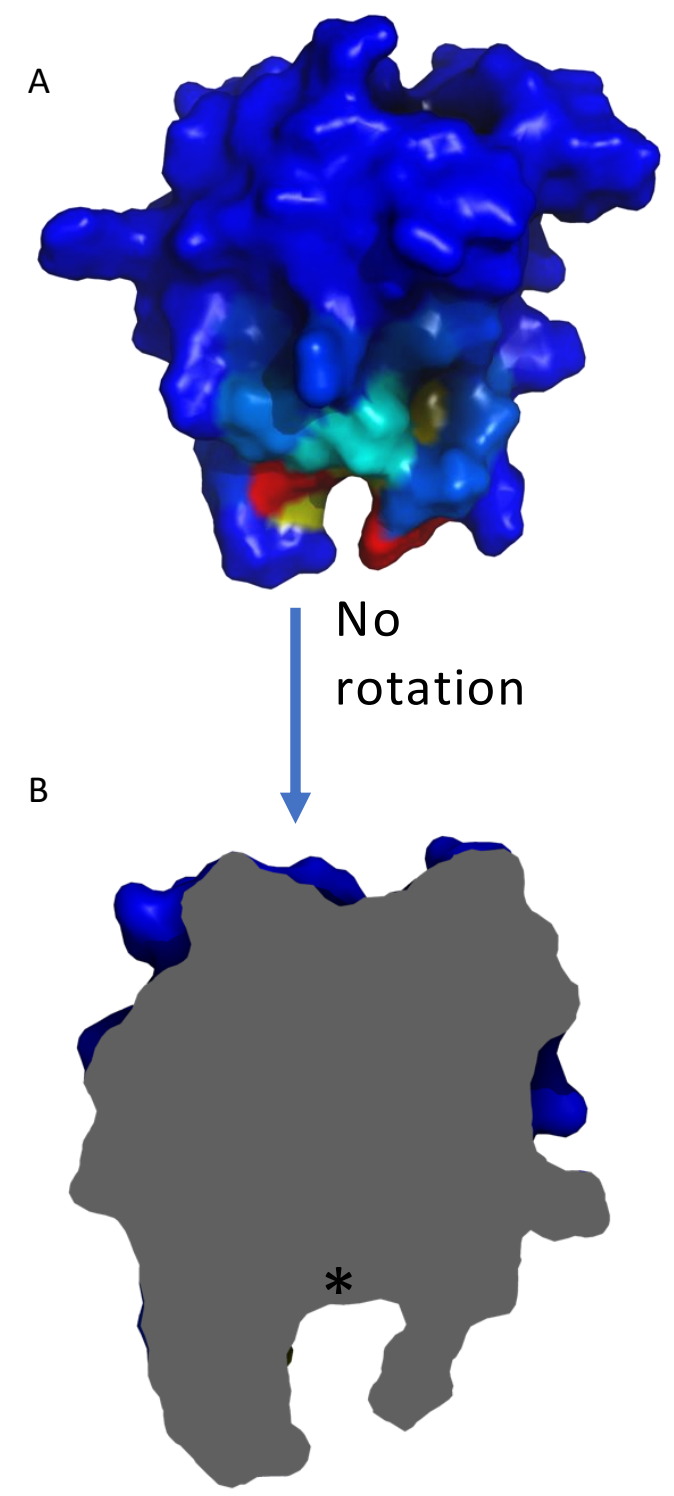
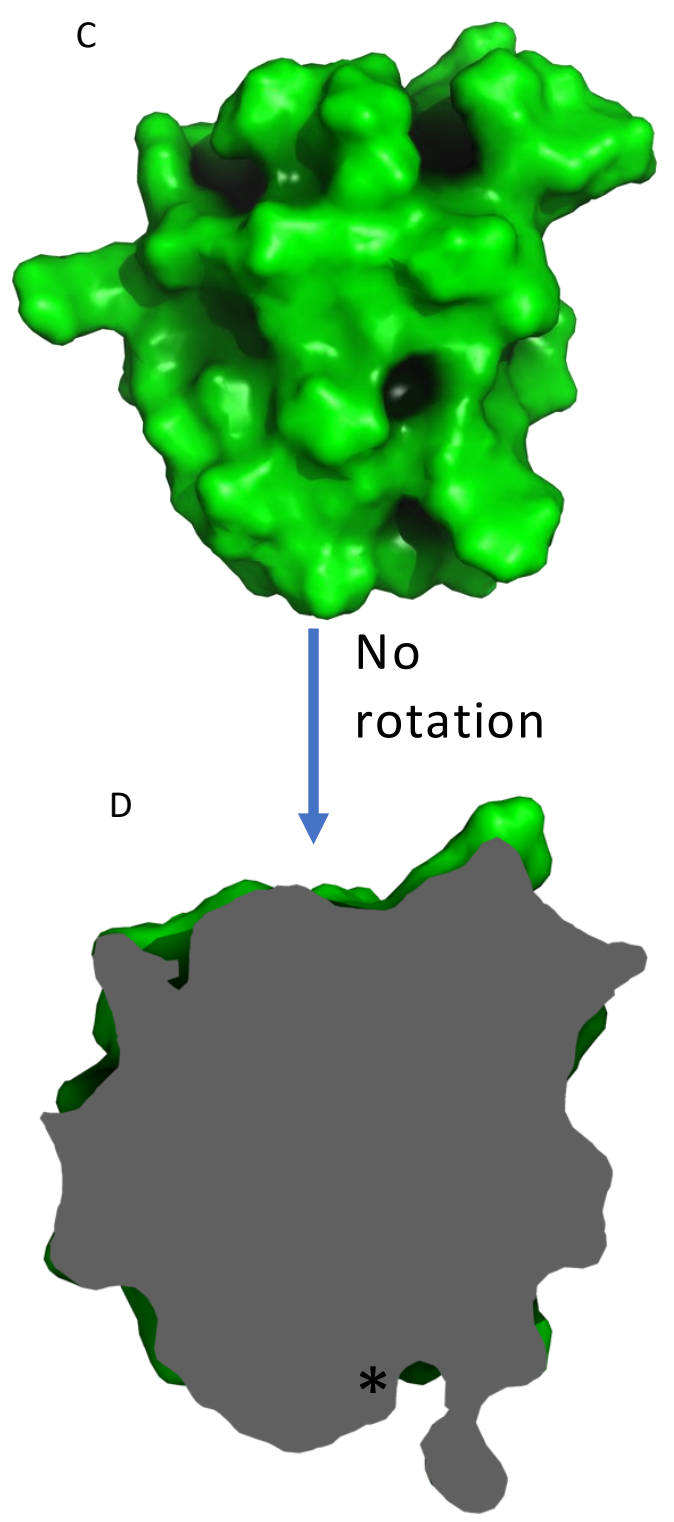

Figure S11. Additional images of the apo-ArCP predicted site shown in Figure $5 \mathrm{E}-\mathrm{F}$. A. Surface representation of Figure 5E. B. Slice views of this frame. The asterisk indicates the predicted site's location. C. Surface view of the first frame of the MD trajectory, aligned with (A). D. Slice view of the first frame. The asterisk indicates the predicted site's location. 

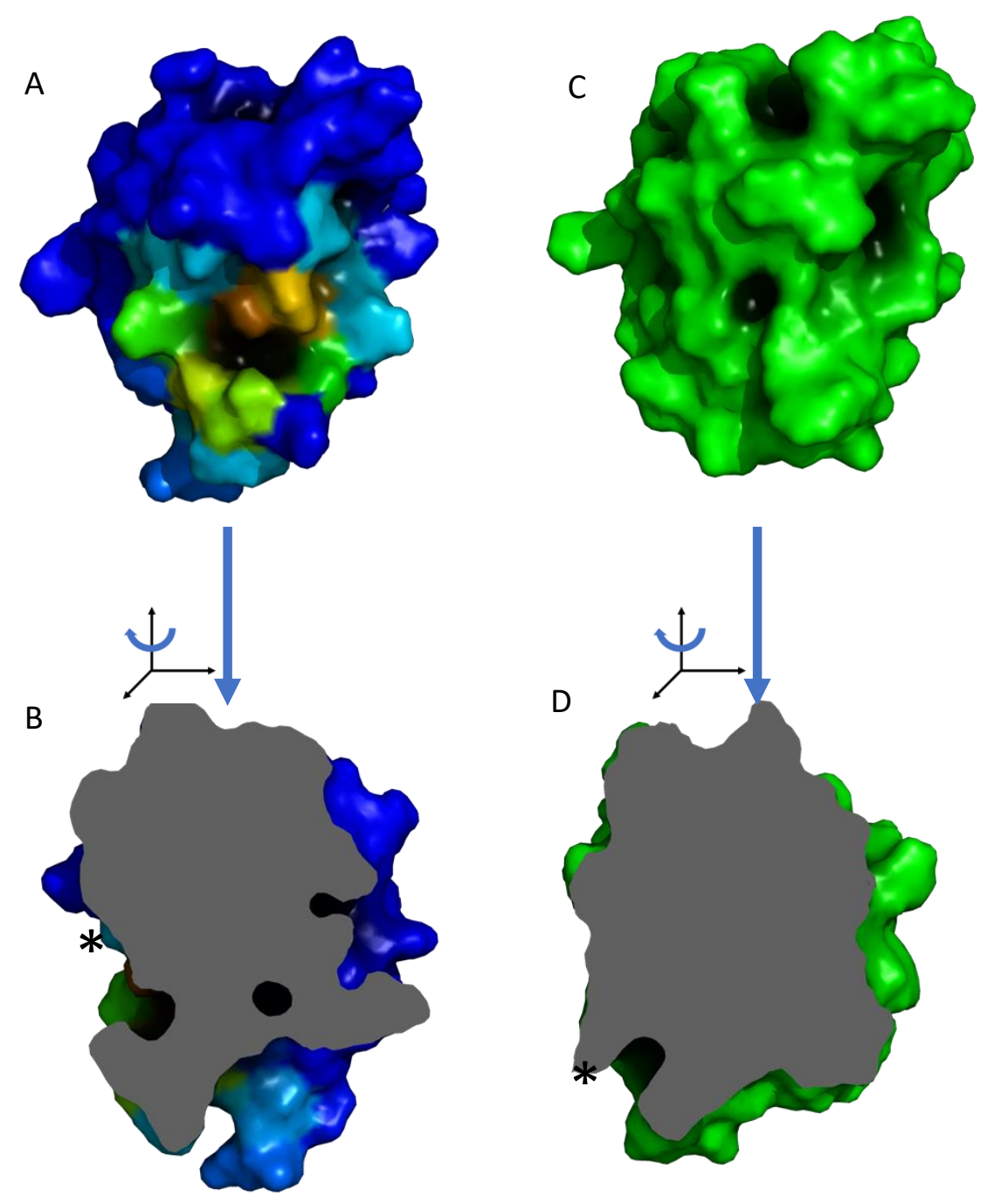

Figure 12. Additional images of the apo-ArCP predicted site shown in Figure $5 \mathrm{G}-\mathrm{H}$. A. Surface representation of Figure 5G. B. Rotated slice view of this conformation. The asterisk indicates the predicted site's location. C. Surface view of the first frame of the MD trajectory, aligned with $(A)$. D. Slice view of the first frame. The asterisk indicates the predicted site's location. 


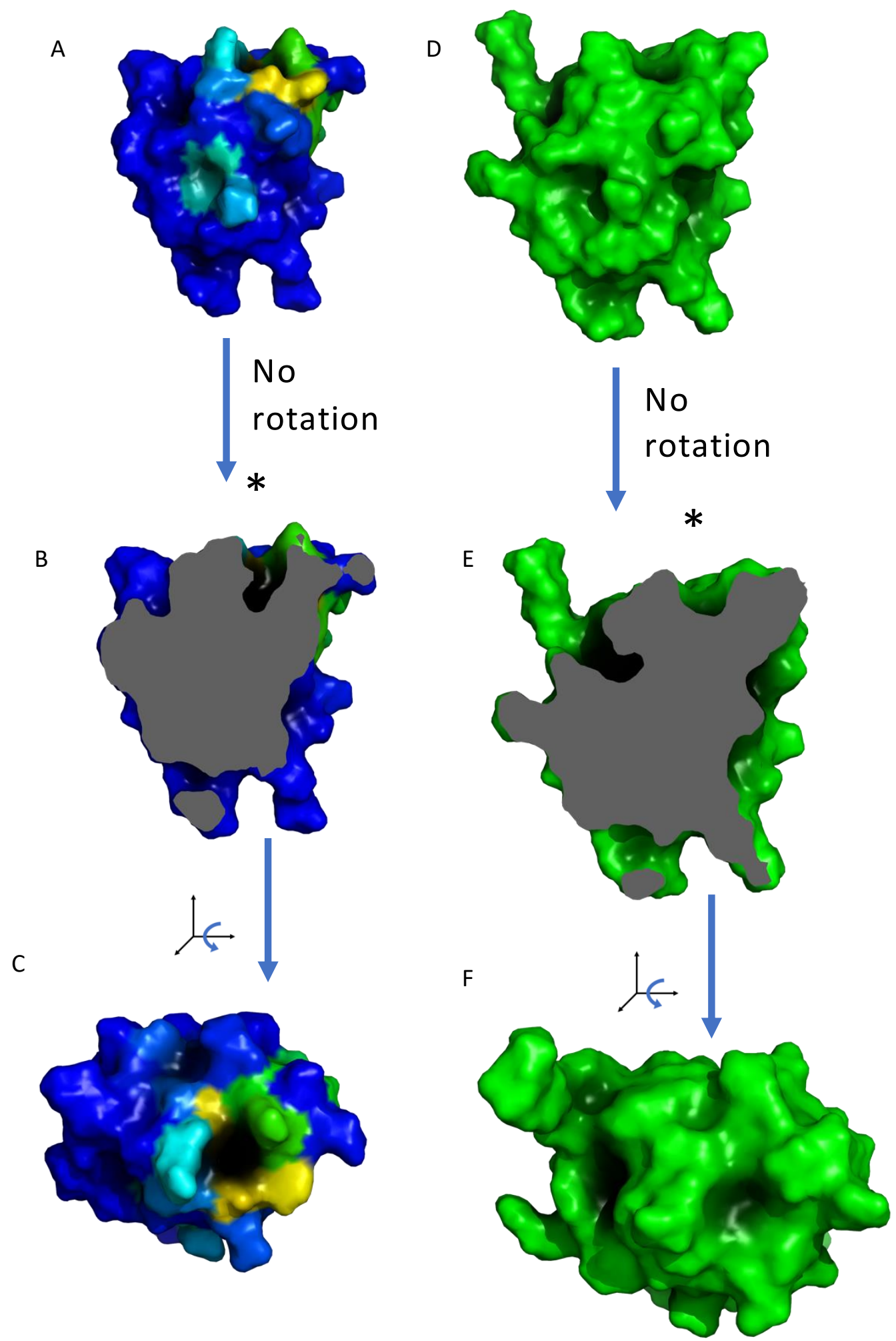

Figure S13. Additional images of a predicted binding site in the holo-ArCP. A. Surface representation of Figure $6 \mathrm{~A}$. B. Slice representation of the site. The asterisk marks the site location. C. Rotated view of the site. D. Surface view of the first frame of the MD trajectory, aligned with (A). E. Slice view of the first frame. The asterisk indicates the predicted site's location. F. Rotated view of the first frame. 

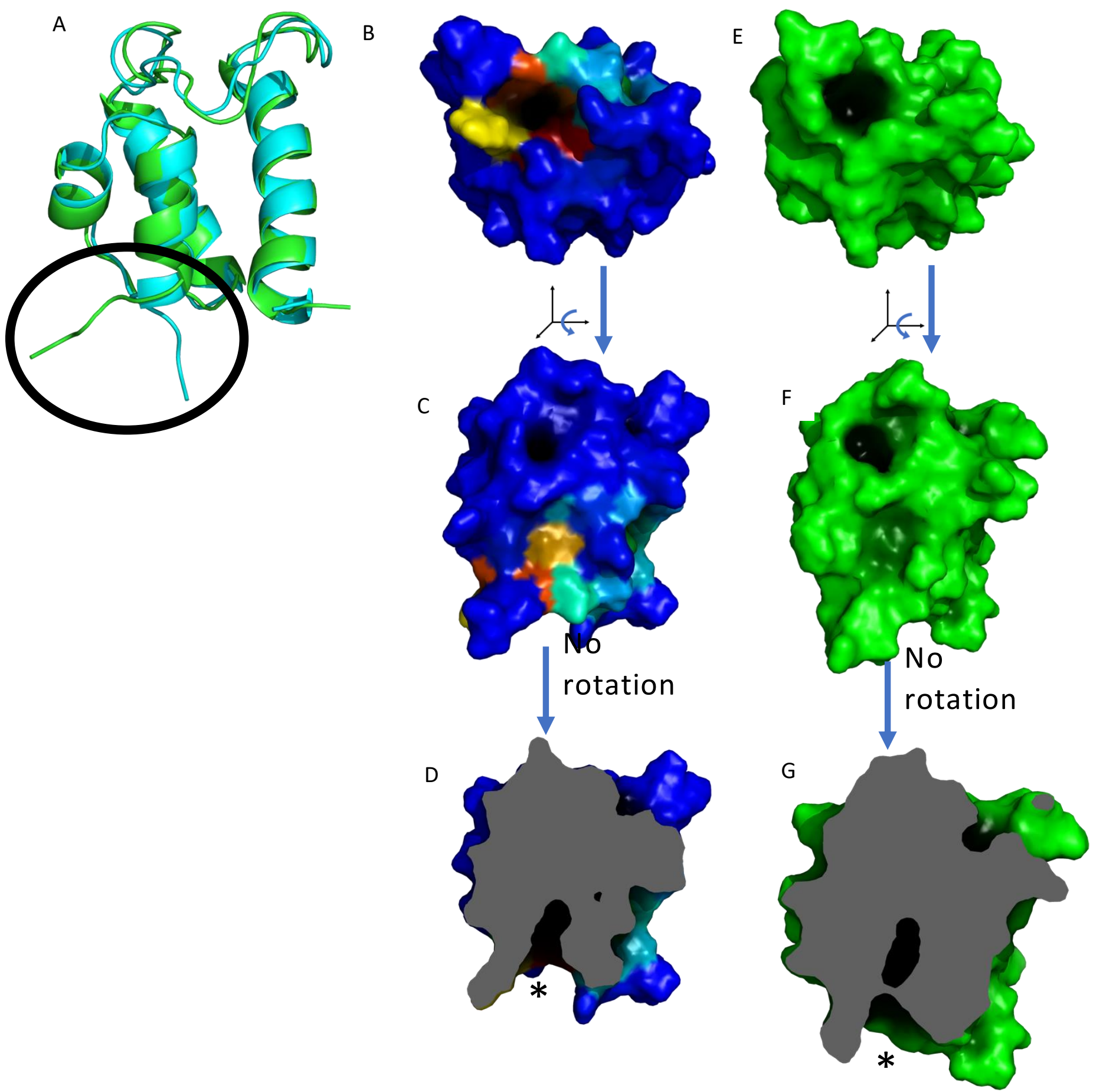

Figure S14. Additional images of a predicted binding site in the loaded-ArCP. A. Alignment between a frame from the apo data (blue) and a frame from the loaded data (green). The $C$ termini are circled to emphasize the dramatic difference in conformation. B. Surface representation of Figure 6D. C. Surface representation of Figure 6C. D. Slice view of the predicted site. The asterisk marks the site location. E. Surface view of the first frame of the MD trajectory, aligned with (B). F. Surface view of the first frame, aligned with (C). G. Slice view of the first frame. The asterisk marks the site location. 

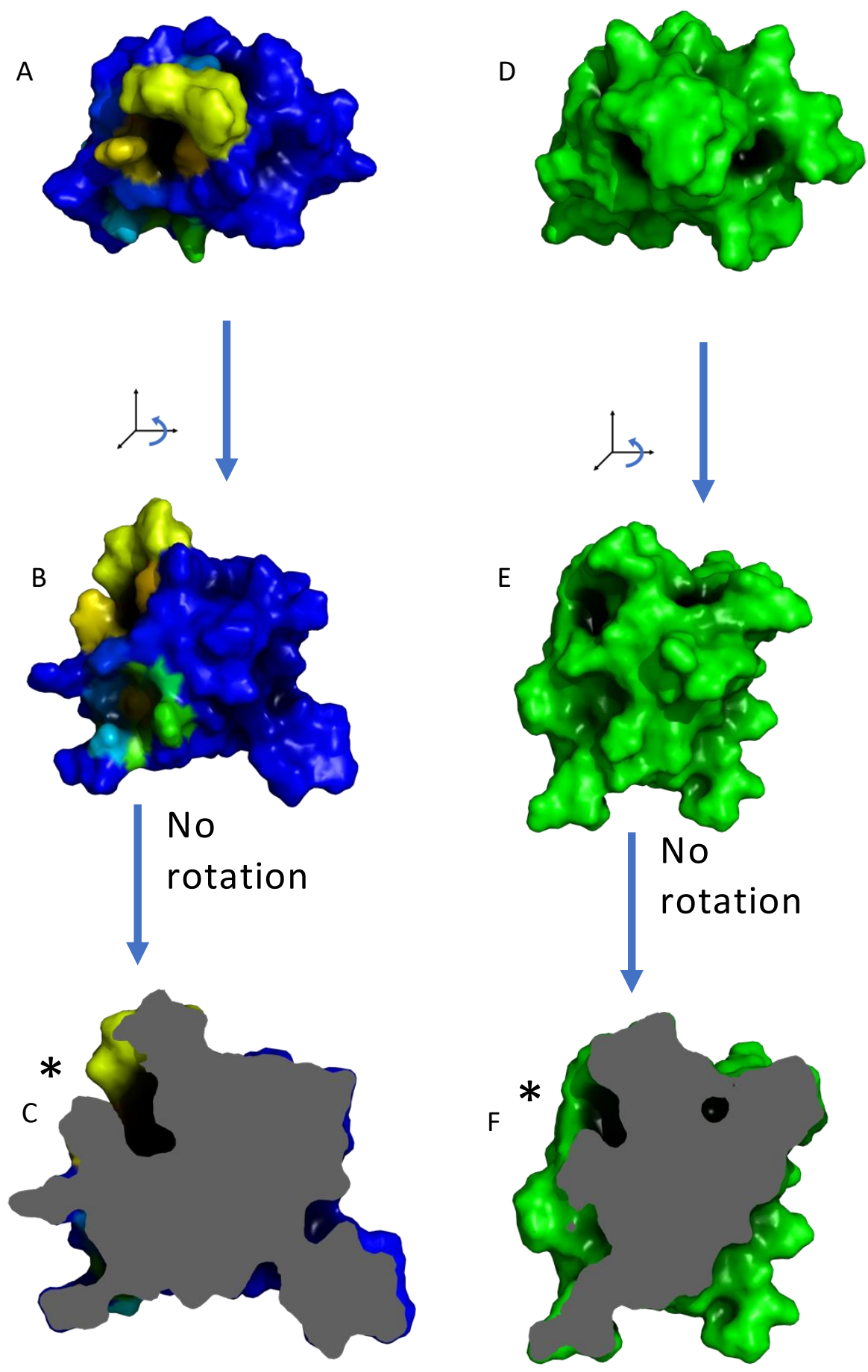

Figure S15. Additional images of a predicted binding site in the loaded-ArCP. In the cartoon representations, the PP arm is shown using blue sticks. On the right, the green proteins are the initial structure (before MD simulation). A. Surface representation of Figure 6F. B. Surface representation of Figure 6E. C. Slice view of the predicted site. The asterisk marks the site location. D. Surface view of the first frame of the MD trajectory, aligned with (A). E. Surface view of the first frame, aligned with (B). F. Slice view of the first frame. The asterisk marks the site location. 

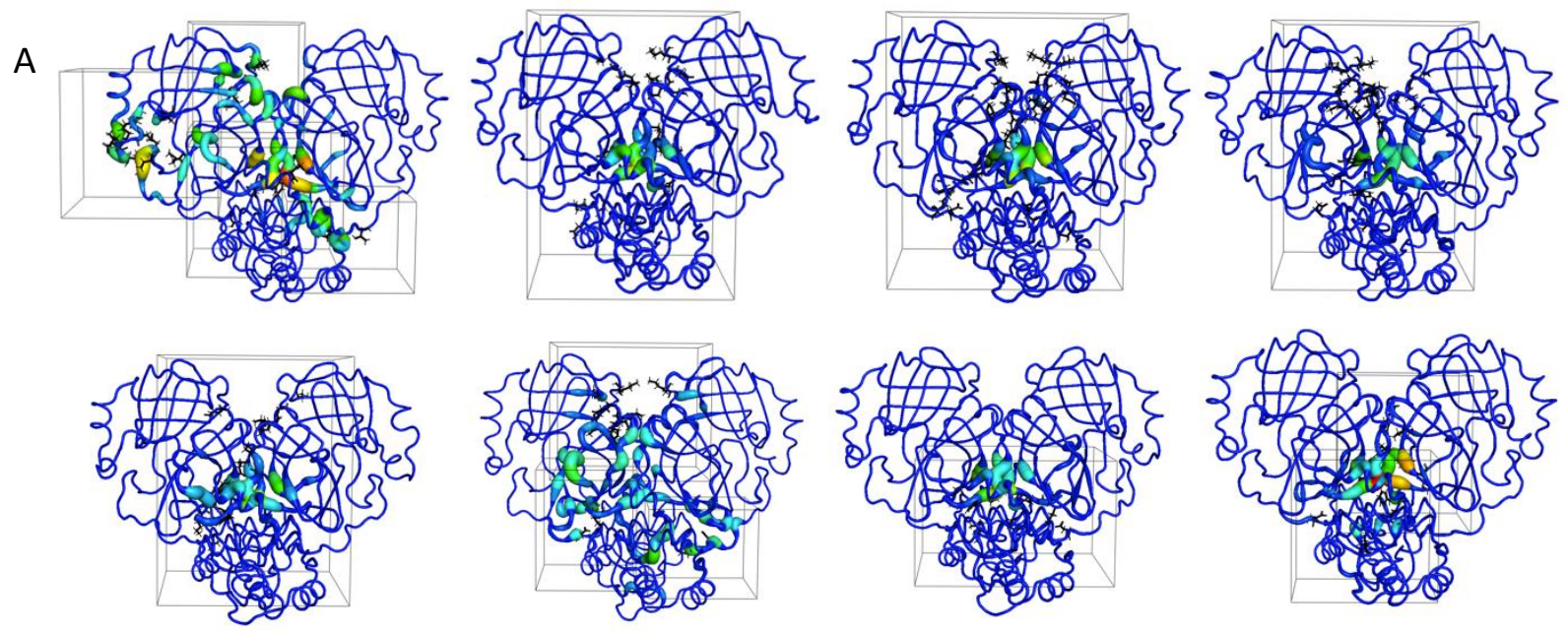

B
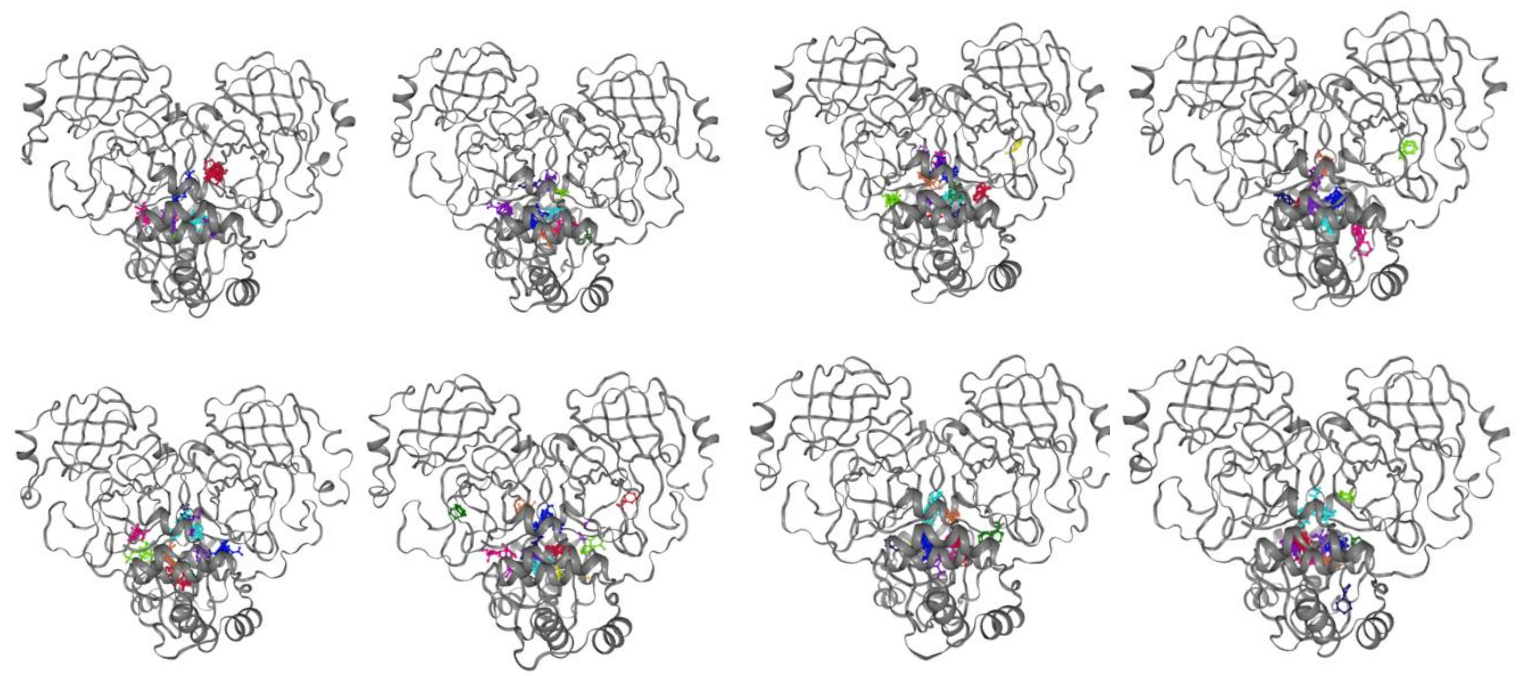

C
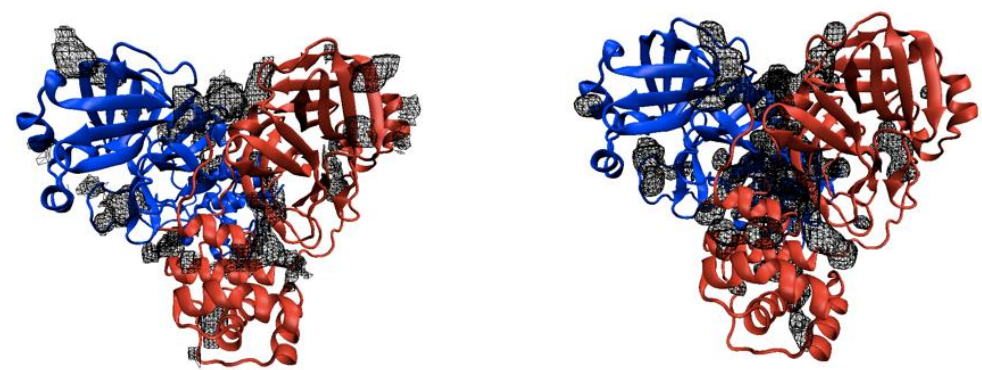

Figure S16: A comparison of pocket predictions for the first 20 ns of SARS-CoV-2 M-Pro. A. Predictions made by TACTICS. B. Predictions made by FTMap for each frame identified by TACTICS's k-means clustering. C. Predictions made by MDPocket. The left shows results when MDPocket was run on the 8 frames shown above; the right shows results when MDPocket was run on every fifth frame of the dataset. Both MDPocket images show the 0.5 frequency isosurfaces. 
Table S1: A list of proteins in the database used to train the TACTICS machine learning model. Each row contains a "holo structure" with a ligand bound at a cryptic site, and a set of "apo structures" of the same protein with no ligand at the cryptic site. Each entry is of the format PDB_CHAIN, i.e. the entry "2ieg_B" is chain B of PDB ID 2IEG. While this database is similar to the CryptoSite database ${ }^{1}$ and extended CryptoSite database ${ }^{2}$, the TACTICS database has several key differences, as discussed in the text. AMPA receptor structures $1 \mathrm{FTL}$ and 1 NOT were removed because we wanted TACTICS to be unbiased for future work involving this protein. The database construction methods resulted in a small number of proteins being included twice; future versions of TACTICS may remove these proteins from the training database.

\begin{tabular}{|c|c|}
\hline $\begin{array}{l}\text { Holo } \\
\text { Structure }\end{array}$ & Apo Structures \\
\hline 2ieg_B & 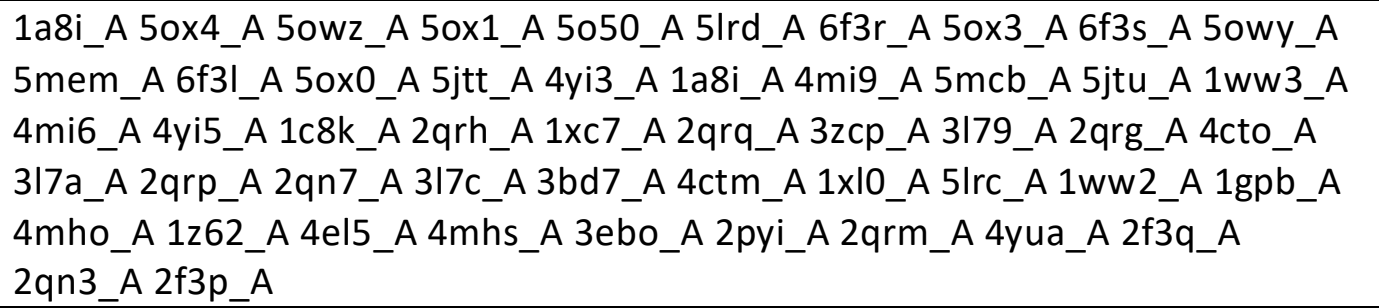 \\
\hline 1cib_A & $\begin{array}{l}\text { 1hoo_B 1ade_A 1ade_A 1ade_B 1hoo_B 1hon_A 1hon_B 1adi_A 1adi_B 1ade_A } \\
\text { 1ade_B 1hoo_B 1hon_A 1hon_B 1adi_A 1adi_B }\end{array}$ \\
\hline 1lic_A & $\begin{array}{l}\text { 1alb_A 1g7n_A 1lib_A 3js1_A 3js1_B 1ab0_A 1a2d_A 1a2d_B 1a18_A 1alb_A } \\
\text { 1acd_A 5c0n_A 5c0n_B 5d8j_A }\end{array}$ \\
\hline 1nx3_A & $\begin{array}{l}\text { 1alv_A 4phj_A 4phj_B 4phn_A 4phn_B 1alv_A 1alv_B 1nx1_A 1nx1_B 1nx2_A } \\
\text { 1nx0_A 1nx0_B 1kfu_S 1kfx_S }\end{array}$ \\
\hline 1kuv_A & 1b6b_A 1b6b_A 1b6b_B \\
\hline 2v5a_A & 1bnc_B 2w6z_B 2v5a_B \\
\hline 1ryo_A & 1bp5_A 1bp5_A 1bp5_B 1bp5_C 1bp5_D 1btj_A 1btj_B \\
\hline 1gx8_A & 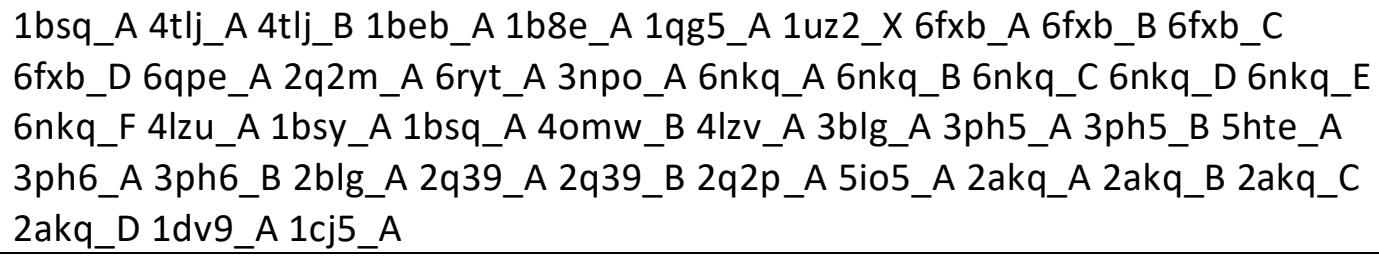 \\
\hline 1ctr_A & 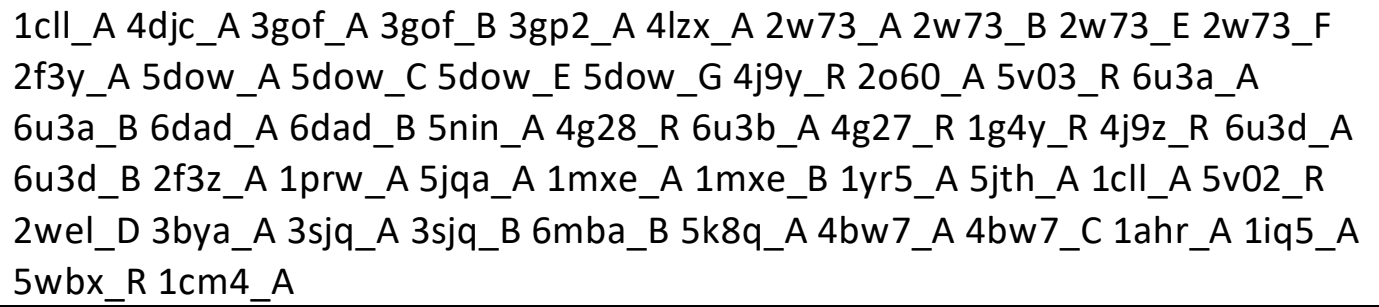 \\
\hline 1ey3_F & 1dub_D 1mj3_D 2dub_C2dub_D 1dub_D \\
\hline 1h9g_A & 1e2x_A 1hw1_A 1hw1_B 1e2x_A 1hw2_A 1hw2_B 1h9t_A 1h9t_B \\
\hline 1ecc_B & 1ecj_D 6czf_A 6czf_B 6czf_C 6czf_D 1ecj_B 1ecj_D 6ott_A 6ott_B \\
\hline 1gky_A & 1ex6_A 1ex6_A 1ex6_B 4f4j_A 4f4j_B \\
\hline
\end{tabular}




\begin{tabular}{|c|c|}
\hline 1ha3_B & $\begin{array}{l}\text { 1exm_A 2c77_A 1exm_A 1eft_A 1ttt_A 1ttt_B 1ttt_C 1tui_A 1tui_B 1tui_C } \\
\text { 1aip_A 1aip_B 1aip_E 1aip_F 1zc8_Y }\end{array}$ \\
\hline $115 s \_B$ & $\begin{array}{l}\text { 1fa9_A 2ati_A 2ati_B 1xoi_A 1xoi_B 3cem_A 3cem_B 1l5r_B 1em6_A 1em6_B } \\
\text { 1fc0_A 1fc0_B 1fa9_A 3ceh_A 3ceh_B 1exv_A } 1 \mathrm{exv} \text { _B 3cej_A 3cej_B 2zb2_A } \\
\text { 2zb2_B }\end{array}$ \\
\hline 2008_X & 1fvr_A 1fvr_A 1fvr_B \\
\hline 3hl8_A & $\begin{array}{l}\text { 1fxx_A 2qxf_A 3c95_A 4hcb_A 4hcb_B 4jrp_A 1fxx_A 3c94_A 4hcc_A 4hcc_B } \\
\text { 4jrq_A 4jrq_B 4js4_A 4js4_B 4js5_A 4js5_B }\end{array}$ \\
\hline 1gzf_C & $\begin{array}{l}\text { 1g24_D 2a78_B 1g24_A 1g24_B 1g24_C 1g24_D 2c89_A 1uzi_A 1uzi_B 2c8g_A } \\
\text { 2c8f_F 2c8f_G 1gze_A 1gze_B 1gze_C 1gze_D }\end{array}$ \\
\hline 2ixu_A & 1h09_A 1h09_A 1oba_A \\
\hline 1ghy_H & 1hag_E 3sqe_E 1hag_E 3sqh_E 1mh0_A 1mh0_B \\
\hline 3ip0_A & $\begin{array}{l}\text { 1hka_A 1tmj_A 3ili_A 1kbr_A 1hka_A 3kue_A 3hsd_B 3ill_A 1im6_A 1g4c_A } \\
\text { 1g4c_B 3hcx_A 3kug_A 2f63_A 2f65_A 1eq0_A }\end{array}$ \\
\hline 1imb_B & 1imf_A 1ime_A 1ime_B 1imf_A \\
\hline 1wun_H & 1jbu_H 1jbu_H 1fak_H \\
\hline 1pzo_A & 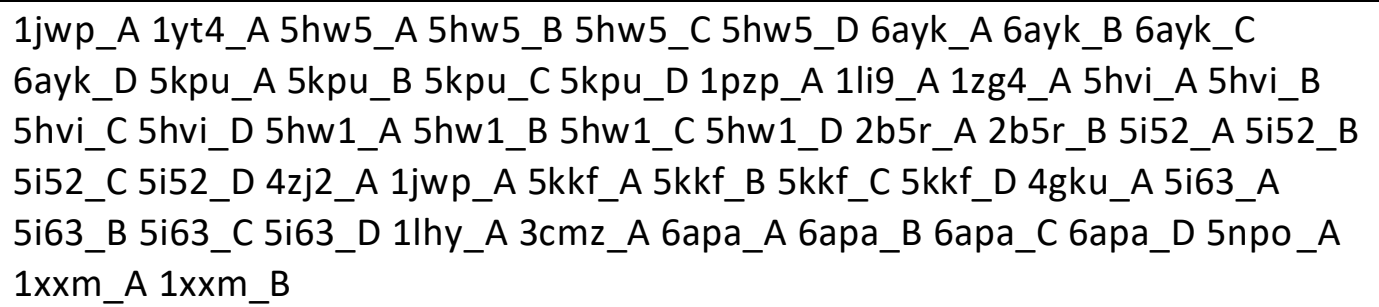 \\
\hline 1u1d_F & 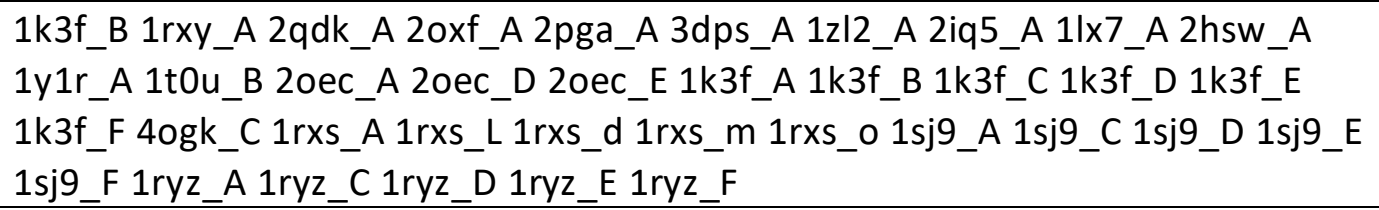 \\
\hline 2egh_B & 1k5h_C 1k5h_A1k5h_C \\
\hline 2ofp_A & 1ks9_A 1ks9_A 1yjq_A \\
\hline 1grn_A & $\begin{array}{l}\text { 1kz7_D 1kz7_B 1kz7_D 6tky_C 3gcg_A 1kzg_B 1kzg_D 6ajl_B 6ajl_D 6ajl_F 6ajl_H } \\
\text { 6aj4_B 6aj4_D 6aj4_F 6aj4_H 5fi1_B 5upl_B 2kb0_A 2ase_A 1ees_A 1aje_A }\end{array}$ \\
\hline 2hka_C & 1nep_A 1nep_A2hka_A \\
\hline 3hok_B & 1ni6_D 1ni6_A1ni6_B 1ni6_C 1ni6_D 1s8c_B 1s8c_C 1s8c_D \\
\hline 1eyj_B & 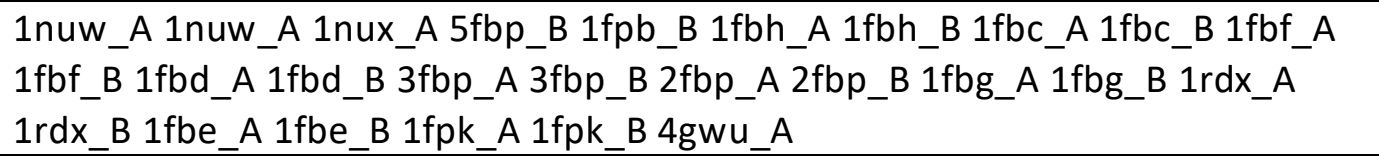 \\
\hline 1oke_B & $\begin{array}{l}\text { 1ok8_A 1ok8_A 3c5x_A 1tg8_A 4uta_A 4uta_B 4ut9_A 4ut9_B 4ut9_C 4ut9_D } \\
\text { 4utc_B 1oan_A 1oan_B 4ut6_A 4ut6_B 4utb_A 4utb_B 5n09_A 5n09_B 3j8d_G } \\
\text { 3j8d_H 3j8d_I3j35_A 3j35_B 3j35_C 3iya_A 3iya_B 3iya_C 3c6r_A 3c6r_B 3c6r_C } \\
\text { 2r6p_A 2r6p_B 2r6p_C 2b6b_A 2b6b_B 2b6b_C 1thd_A 1thd_B 1thd_C 1tge_A } \\
\text { 1tge_B 1tge_C }\end{array}$ \\
\hline 1pzy_D & 1pzt_A 1pzt_A 1nwg_B 1fgx_A \\
\hline $2 w k w \_B$ & 1qlw_B 1qlw_B \\
\hline 3f82_A & 1r1w_A 3q6u_A 1r1w_A 2g15_A \\
\hline
\end{tabular}




\begin{tabular}{|c|c|}
\hline $1 j 6 z \_A$ & $\begin{array}{l}\text { 1rdw_X 2q0u_A 2pbd_A 3mn5_A 2q0r_A 3sjh_A 4pkg_A 2pav_A 6jh9_A 2a40_A } \\
\text { 2a40_D 4b1x_B 2a42_A 2gwj_A 3w3d_A 5yee_B 4h03_B 1t44_A 6rsw_A } \\
\text { 4b1w_B 2gwk_A 2gwk_B 4pl8_A 4pl8_B 2a3z_A 3u9z_A 3u8x_A 3u8x_C 5ypu_A } \\
\text { 5ypu_C 4b1u_B 6jh8_A 2ff3_B 2ff6_A 1rdw_X 1esv_A 4pki_A 1eqy_A 1kxp_A } \\
\text { 6jcu_A 6jcu_C 1mdu_B 1mdu_E 4h0t_B 2v51_B 2v51_D 4z94_A 2a41_A 6jbk_A } \\
\text { 6jbk_C 6jbk_E }\end{array}$ \\
\hline $2 w 5 k \_B$ & $\begin{array}{l}\text { 1rhb_A 6etl_A 6etk_A 6etm_A 6etn_A 6eto_A 6etp_A 6etq_A 1kf5_A 1kf7_A } \\
\text { 1kf8_A 6f60_B 6etr_A 2rat_A 3rat_A 4rat_A 5rat_A 6rat_A 1eic_A 8rat_A 7rat_A } \\
\text { 1eid_A 1fs3_A 1ymn_A 1eie_A 1rat_A 1rhb_A 2g8q_A 2g8q_B 9rat_A 1izr_A } \\
\text { 1izp_A 1ymr_A 1ymw_A 3rsd_A 3fl3_B 2p43_A 3d6o_B 3d6q_B 3d7b_B 3d6p_B } \\
\text { 2op2_A 1rbx_A 5e5f_B 1c9v_A 4wyn_A 4wyn_B 4l55_A 4l55_B 1rbw_A 4g8v_B }\end{array}$ \\
\hline 1s9d_A & $\begin{array}{l}\text { 1rrg_A 1r8s_A 103y_A 103y_B 1j2j_A 6ii6_C 6ii6_D 1hur_A 1hur_B 2j59_A } \\
\text { 2j59_B 2j59_C 2j59_D 2j59_E 2j59_F 1rrg_A 1rrg_B 4c0a_C 4c0a_D 4c0a_G } \\
\text { 4hmy_C 6cri_C6cri_H 6cri_K 6cri_L 6cri_U 6cri_V 1u81_A }\end{array}$ \\
\hline 1br6_A & 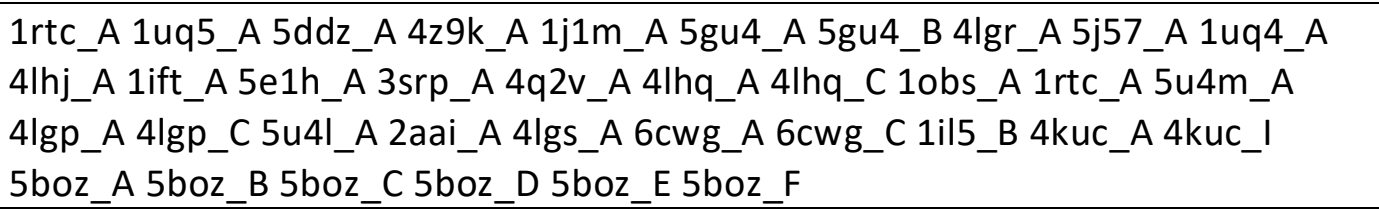 \\
\hline 3fgo_B & 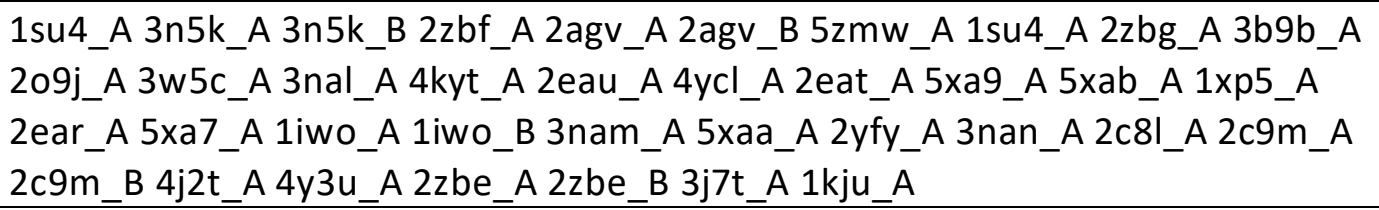 \\
\hline 2eum_A & 1swx_A 1wbe_A 3rwv_A 3rwv_B 1tfj_A 1swx_A 2evt_A \\
\hline 1tr5_A & 1tqo_A 1tqo_A \\
\hline $2 g z 7 \_A$ & $\begin{array}{l}\text { 1uk2_A 2h2z_A 2qcy_A 2duc_A 2duc_B 2gt7_B 3snd_A 3snd_B 1q2w_A 2gt8_A } \\
\text { 2bx3_A 2c3s_A 1uj1_A 1uj1_B 4hi3_A 4hi3_B 3aw1_A 3aw1_B 3vb3_A 3vb3_B } \\
\text { 3ea8_A 5b6o_A 5b6o_B 2gz9_A 3m3s_A 3m3s_B 3f9f_A 3f9f_B 3ea9_A 3fzd_A } \\
\text { 3aw0_A 1uk2_A 1uk2_B 3e91_A 3e91_B 3snb_A 2q6g_A 2q6g_B 3sne_A 3ea7_A } \\
\text { 3ea7_B 3avz_A 1uk3_A 1uk3_B 3m3v_A 3m3v_B 3snc_A 3eaj_A 3eaj_B 2qc2_A } \\
\text { 2qc2_B }\end{array}$ \\
\hline 3ixj_C & $\begin{array}{l}\text { 1w50_A 3tpj_A 5mcq_A 1fkn_A 1fkn_B 1xn2_A 1xn2_B 1xn2_C 1xn2_D 1w50_A } \\
\text { 1sgz_A 1sgz_B 1sgz_C 1sgz_D 1xn3_A 1xn3_B 1xn3_C 1xn3_D 3159_B 2zhv_A } \\
\text { 1m4h_A 1m4h_B 2zht_A 2zhr_A 2zhr_B 2zhu_A 3tpl_A 3tpl_B 3tpl_C 5mbw_A } \\
\text { 2zhs_A 3r1g_B }\end{array}$ \\
\hline 10w3_B & $\begin{array}{l}\text { 1xcg_B 5jhg_F 1xcg_B 1xcg_F 3t06_B 3t06_F 1lb1_B 1lb1_D 1lb1_F 1lb1_H } \\
\text { 2rgn_C 2rgn_F }\end{array}$ \\
\hline 1Xvc_A & 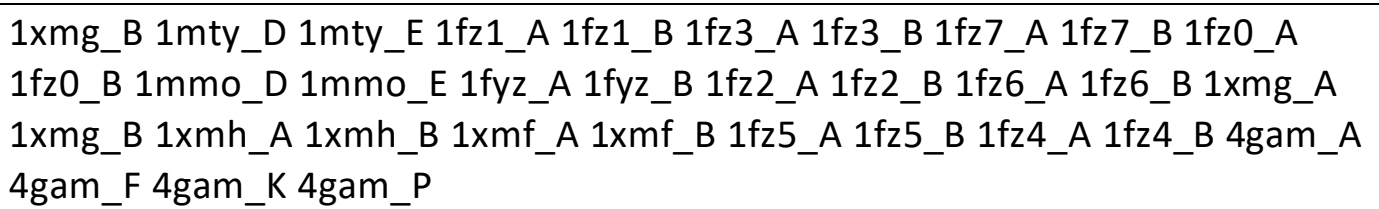 \\
\hline 1py2_A & $\begin{array}{l}\text { 1z92_A 5lqb_A 4nem_A 1m47_A 1nbp_A 2b5i_A 3ink_C 3ink_D 5utz_A 5utz_D } \\
\text { 5utz_E 5utz_I 1m4c_A 1m4c_B 1z92_A 2erj_D 2erj_H 3qb1_A 3qb1_B 3qb1_C }\end{array}$ \\
\hline
\end{tabular}




\begin{tabular}{|c|c|}
\hline & $\begin{array}{l}\text { 3qb1_E 3qb1_F 3qb1_H 3qaz_A 3qaz_D 3qaz_G 3qaz_J 3qaz_M 3qaz_P 3qaz_S } \\
\text { 3qaz_V 3qaz_Y 3qaz b 3qaz e 3qaz h } 1 \mathrm{irl} A\end{array}$ \\
\hline 2ot1_D & 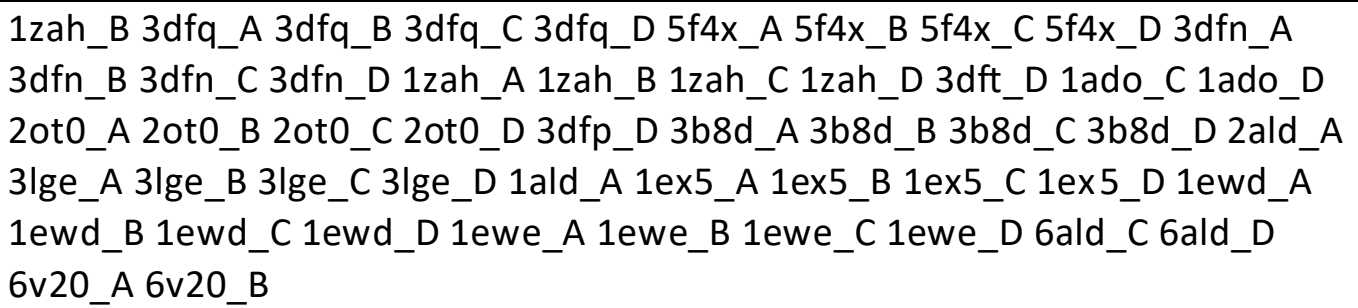 \\
\hline 1za1_D & $\begin{array}{l}\text { 2air_H 1d09_B 1d09_D 2air_B 2air_H 2qgf_B 2qgf_D 4wto_B 4wto_D 1f1b_B } \\
\text { 1f1b_D 1nbe_B 1nbe_D 1tu0_B 1tu0_D 1i5o_D 1r0c_B 1r0c_H 1xjw_B 1xjw_D } \\
\text { 1tth_B 1tth_D 1ezz_B 1ezz_D 2qg9_B 2qg9_D 1q95_G 1q95_H 1q95_I 1q95_J } \\
\text { 1q95_K 1q95_L 3d7s_B 3d7s_D 2ipo_B 2ipo_D 1r0b_G 1r0b_H 1r0b_I 1r0b_J } \\
\text { 1r0b_K 1r0b_L }\end{array}$ \\
\hline 1yv3_A & 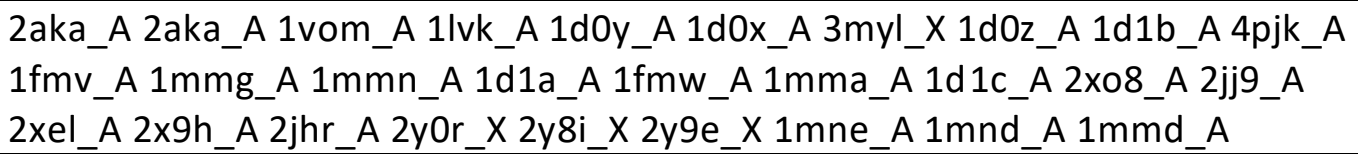 \\
\hline 2piq_A & 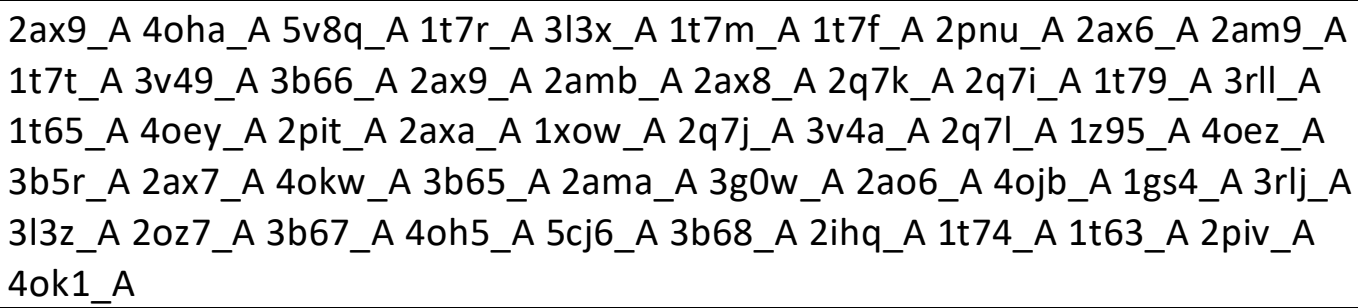 \\
\hline 3dhh_E & $\begin{array}{l}\text { 2bf3_A 3ge3_E 5tdu_E 2bf5_A 2bf5_B 3dhi_E 5tdt_E 5tdt_H 3q3n_E 3i5j_E } \\
\text { 3q3o_E 3q2a_E 5tdv_E 5tdv_H 3i63_E 3ri7_E 3ge8_E 3ge8_H 2bf2_A 2bf2_B } \\
\text { 1g10_A 1g11_A }\end{array}$ \\
\hline 3gqz_A & 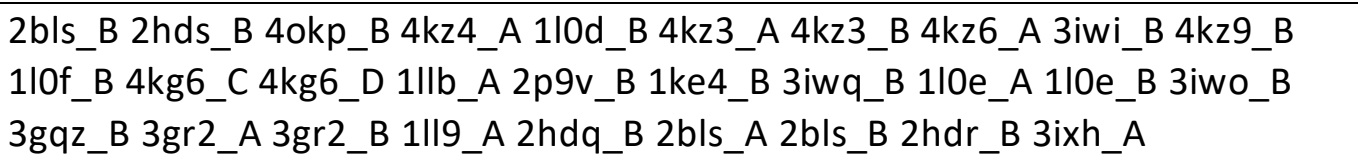 \\
\hline 2gir_B & 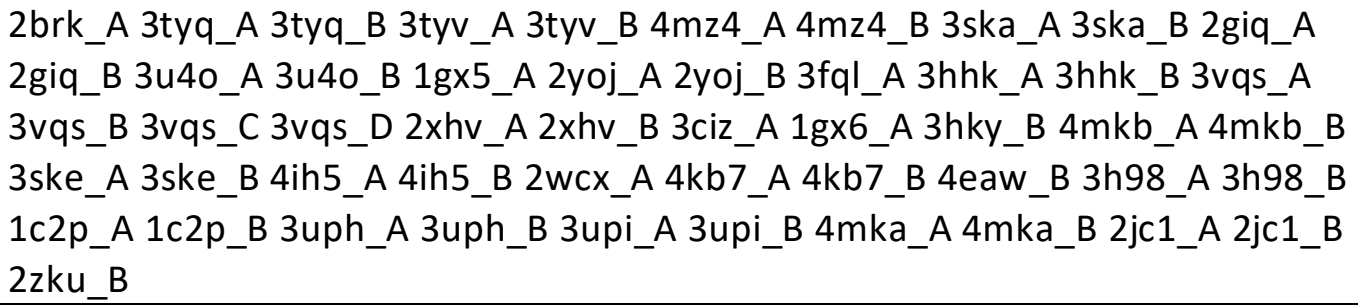 \\
\hline 2bu2_A & $\begin{array}{l}\text { 2bu8_A 4mpc_A 4mpn_A 4mp2_A 4mp7_A 4mpe_A 5j6a_A 5m4p_A 3crk_A } \\
\text { 3crk_B 4v26_A 2bu7_A 1jm6_A } 1 \mathrm{jm} 6 \text { _B 2btz_A 4v25_A 5m4n_A 5m4k_A } \\
\text { 2bu8_A 3crl_A 3crl_B }\end{array}$ \\
\hline 1afq_c & $\begin{array}{l}\text { 2cga_B 1t8o_A 1t8o_C 1t8l_A 1t8l_C 2cga_A 2cga_B 1t8n_A 1p2m_A 1p2m_C } \\
\text { 1p2n_A 1p2n_C 1t8m_A 1t8m_C 1p2q_A 1p2q_C 1t7c_A 1t7c_C 1acb_E 3t62_C } \\
\text { 1gl1_A 1gl1_B 1gl1_C 5j4s_A 1p2o_A 1p2o_C 1oxg_A 1cgi_E 1cgj_E 5j4q_A } \\
\text { 1glo_E 1ex3_A 2y6t_A 2y6t_B 2y6t_C 2y6t_D 1chg_A }\end{array}$ \\
\hline 2h4k_A & $\begin{array}{l}\text { 2cm2_A 5qgf_A 2cm2_A 5qgb_A 5qdk_A 5qfe_A 5qf3_A 5qgc_A 5qfk_A 5qfh_A } \\
\text { 5qef_A 1pa1_A 5qdq_A 5qdi_A 5qfu_A 5qfq_A 5qg8_A 5qg3_A } 5 q f r \_A ~ 5 q f v \_A\end{array}$ \\
\hline
\end{tabular}




\begin{tabular}{|c|c|}
\hline & 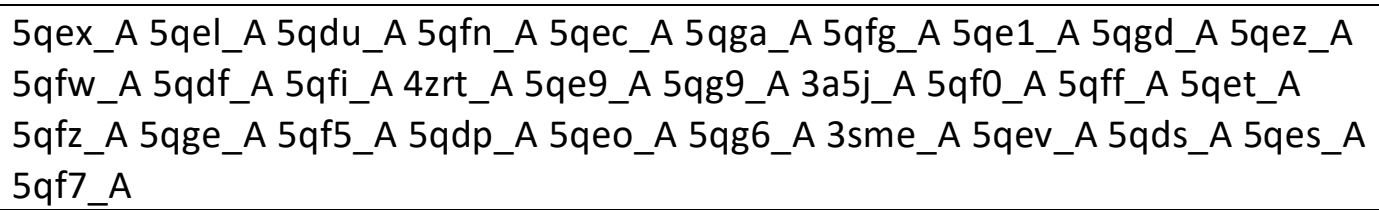 \\
\hline 1t49_A & 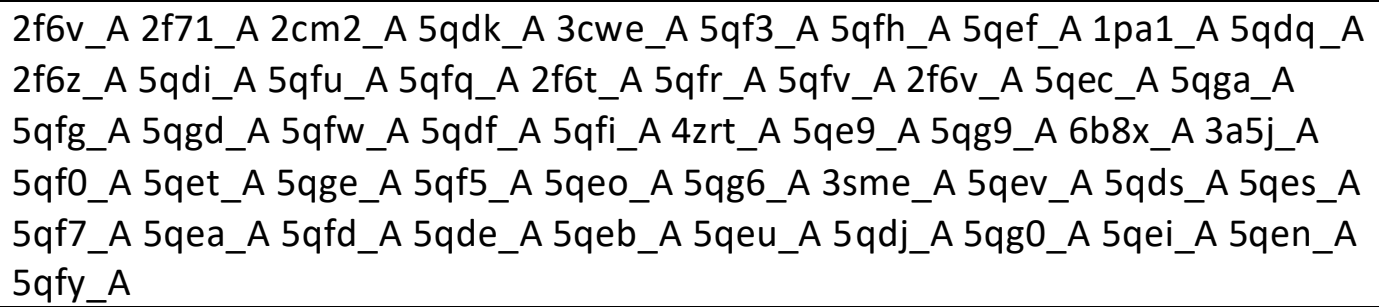 \\
\hline 2jds_A & 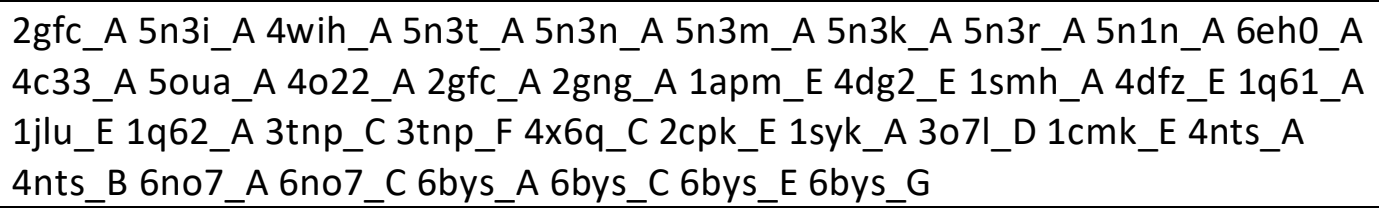 \\
\hline 1s9q_B & $\begin{array}{l}\text { 2gpo_A 6i65_A 2e2r_A 6i66_A 6i62_A 6i61_A 6i67_A 2zkc_A 2zbs_A 2gpo_A } \\
\text { 6i64_A 6i63_A 2zas_A 2p7g_A 1s9p_A 1s9p_B 1s9p_C 1s9p_D 2p7a_A 2gp7_A } \\
\text { 2gp7_B 2gp7_C 2gp7_D 1tfc_A 1tfc_B 2gpp_A 2gpp_B 1kv6_A 1kv6_B }\end{array}$ \\
\hline $3 c f n \_B$ & 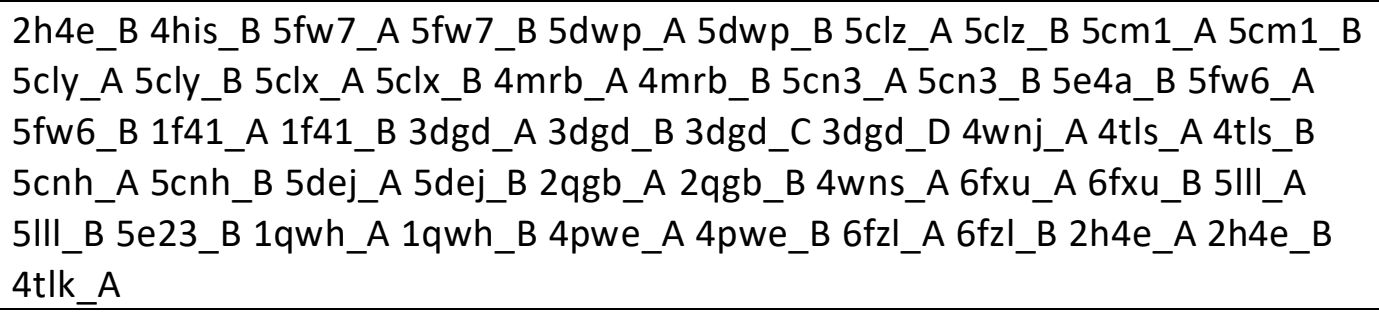 \\
\hline 2iyq_A & 2iyt_A 2iyt_A 2iyt_A \\
\hline 2ohv_A & 2ohg_A 2ohg_A \\
\hline $2 q 8 h \_A$ & 2q8f_A 2q8g_A 2q8f_A \\
\hline 2wi7_A & 2qfo_B 3t0h_A 5j80_A 5j2v_A 1yer_A 2qfo_B 3b26_B 1yes_A 2yeg_A 2k5b_A \\
\hline 3dc1_A & 2qlr_C 6d0a_A 5efs_A 5eun_A 5tf5_B 2qlr_A 2qlr_B 2qlr_C 2qlr_D \\
\hline $2 v 57 \_A$ & 2wgb_A 2v57_B 2v57_D 2wgb_A \\
\hline 1d6y_B & $\begin{array}{l}\text { 2wgq_B 6grr_A 6grr_B 6ezz_A 6ezz_B 1oac_A 1oac_B 1qak_A 1qak_B 1dyu_A } \\
\text { 1dyu_B 1spu_A 1spu_B 2wof_A 2wof_B 1qaf_A 1jrq_A 1jrq_B 1qal_A 1qal_B } \\
\text { 2wo0_A 2wo0_B 2wgq_A 2wgq_B 2woh_A 2woh_B }\end{array}$ \\
\hline 2yqs_A & 2yqc_A 2yqc_A \\
\hline 2npq_A & $\begin{array}{l}\text { 2zb1_A 6sp9_A 6sov_A 6spl_A 5lar_A 6sou_A 6so4_A 6sfi_A 6so2_A 6sot_A } \\
\text { 6soi_A 6so1_A 3fmk_A 2rg6_A 6m95_A 6sfo_A 3gc7_A 6sfk_A 2gfs_A 3kq7_A } \\
\text { 1wbs_A 3nnw_A 2zaz_A 3zya_A 2gtn_A 3d83_A 6sod_A 5xyy_A 4aa0_A 4lop_A } \\
\text { 4lop_B 4lop_D 3fl4_A 2qd9_A 2y8o_A 3fly_A 3e93_A 6sfj_A 3e92_A 4e5a_X } \\
\text { 3s3i_A 4kin_A 4kin_B 4kin_C 4kin_D 3fmn_A 2i0h_A 1wbv_A 5uoj_A 3fln_C } \\
\text { 4loo_A }\end{array}$ \\
\hline 2al4_F & 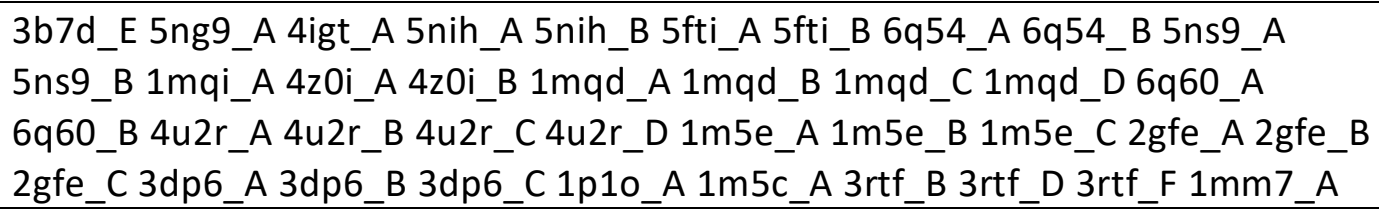 \\
\hline
\end{tabular}




\begin{tabular}{|c|c|}
\hline & $\begin{array}{l}\text { 1mm7_B 1mm7_C 4o3a_B 1wvj_A 1mqj_A 1m5d_A 1ftk_A 4h8j_A 4h8j_B } \\
\text { 4h8j_C 4h8j_D 1syh_A }\end{array}$ \\
\hline 3bl7_A & 3bl9_B 3bl9_B 3bl7_B \\
\hline 2iuz_B & 3che_A 1w9p_A 1w9p_B 1wno_A 1wno_B \\
\hline $3 f q k \_B$ & $\begin{array}{l}\text { 3cj0_A 1gx5_A 4eo6_A 4eo6_B 4eo8_A 4eo8_B 3frz_A 3cj2_A 3cj2_B 2d3z_A } \\
\text { 2d3z_B 2xhv_A 3ciz_A 3ciz_B 1gx6_A 3cj0_A 3cj0_B 4jju_A 4jju_B 2wcx_A } \\
\text { 2gir_A 2gir_B 2wrm_A 5czb_A 5czb_B 1c2p_A } 1 \mathrm{c} 2 \mathrm{p} \_B \text { 3cj5_A 3cj5_B 3cj3_A } \\
\text { 3cj3_B 3mf5_A 3mf5_B 2zku_A 2zku_B 2zku_C 2zku_D 4j02_A 4j02_B 1nb4_A } \\
\text { 1nb4_B 4j04_A 4j04_B 4j06_A 4j06_B 2d3u_A 2d3u_B 4dru_A 2who_A 2who_B } \\
\text { 1nhu_A }\end{array}$ \\
\hline 3bqm_c & $\begin{array}{l}\text { 3f74_C 1mjn_A 3f74_A 3f74_B 3f74_C 1lfa_A 1lfa_B 1zon_A 1zop_A 1zop_B } \\
\text { 3bn3_A 3hi6_A 3hi6_B 3eoa_I 3eoa_J 3tcx_B 3tcx_D 3tcx_F 3tcx_H 3tcx_J 3tcx_L } \\
\text { 3tcx_N 3tcx_P 3tcx_R 3tcx_T 3tcx_V 3tcx_X 3tcx_Z 3tcx_b 1zoo_A 1zoo_B } \\
\text { 1mq8_B 1mq8_D 3eob_I 3eob_J 1dgq_A }\end{array}$ \\
\hline 2yxj_A & $\begin{array}{l}\text { 3fdl_A 3fdl_A 2yq7_A 5vx3_A 5vx3_C 5vx3_E 5vx3_G 3r85_A 3r85_B 3r85_C } \\
\text { 3r85_D 4z9v_A 4z9v_B 3fdm_C 5b1z_A 5b1z_B 2yj1_C 6dco_A 6dco_B 3io8_A } \\
\text { 3io8_C 6dcn_A 6dcn_B 4a1w_B 4a1w_D 5c3g_A 2p1l_A 2p1l_C 2p1I_E 2p1I_G } \\
\text { 4cin_A 4cin_B }\end{array}$ \\
\hline 2wcg_A & $\begin{array}{l}\text { 3gxd_B 3gxi_A 3gxi_B 3gxi_C 3gxi_D 1ogs_B 2nt1_A 2nt1_B 2nt1_C 2nt1_D } \\
\text { 3rik_A 3rik_C 3gxm_A 3gxm_B 3gxm_C 3gxm_D 2f61_A 2f61_B 3ke0_A 2wkl_A } \\
\text { 2wkl_B 3gxd_A 3gxd_B 3gxd_C 3gxd_D 3keh_A 2j25_A 2j25_B }\end{array}$ \\
\hline 3h9j_D & 3h5r_A 3h5r_C 3h5r_D 3h9q_A 3h9q_C 3h9q_D 3h5a_A 3h5a_B 3h5a_C 3h5a_D \\
\hline 1q0b_B & $\begin{array}{l}\text { 3hqd_A 1ii6_A 1ii6_B 3hqd_A 3hqd_B 4zhi_A 4zhi_B 4ck5_C 4ck6_C 4ck7_C } \\
\text { 4aqv_C 4aqw_c }\end{array}$ \\
\hline 3Ith_A & $\begin{array}{l}\text { 3kqa_B 1naw_A 1naw_B 4e7f_D 3kqa_A 3kqa_B 3kqa_C 3kqa_D 4e7e_B 3spb_A } \\
\text { 3spb_B 3spb_C 3spb_D 4e7d_B 4e7d_C }\end{array}$ \\
\hline 2hvd_C & 317u_C 317u_A 317u_C 1jxv_A 1jxv_B 1jxv_C 1jxv_D 1jxv_E 1jxv_F \\
\hline 3eks_A & 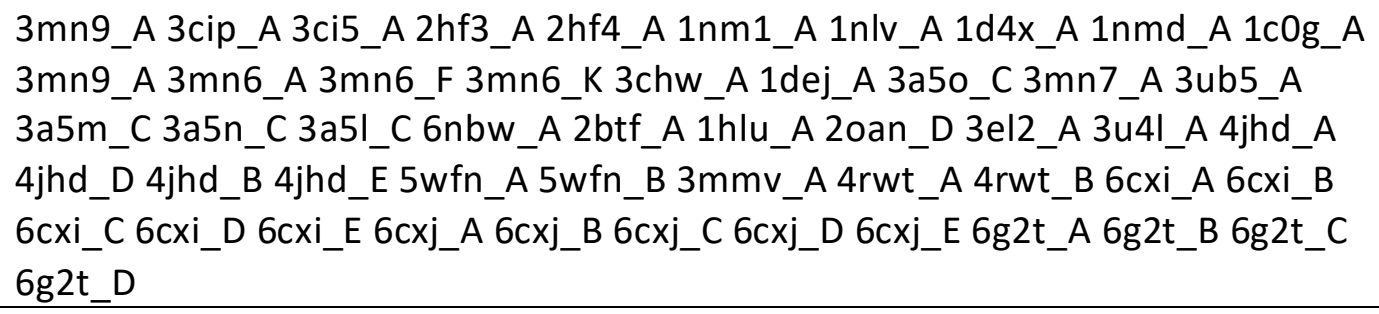 \\
\hline 3hl7_A & 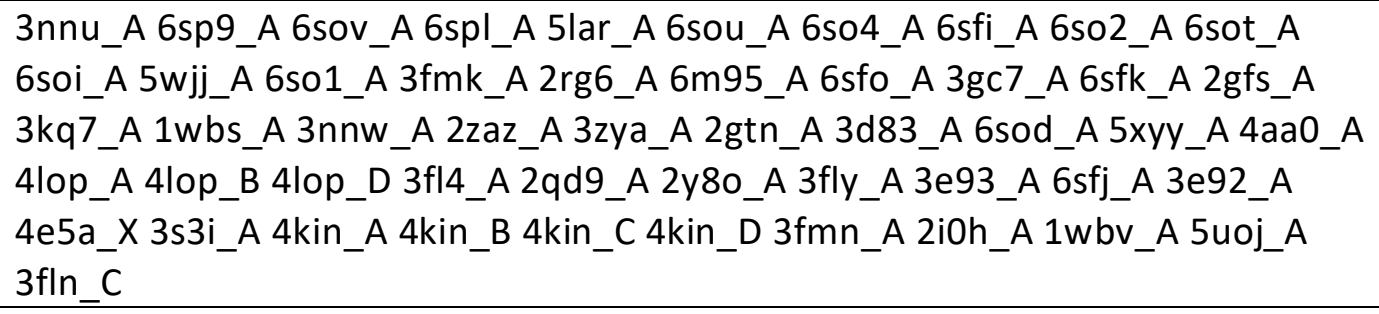 \\
\hline 2bys_J & $\begin{array}{l}\text { 3peo_G 4zk4_B 4zk4_D 2y7y_E 4wv9_A 4wv9_D 2xyt_A 2xyt_C 2xyt_E 2xyt_I } \\
\text { 2xyt_J 2byp_A 2byp_B 2byp_C 2byp_D 2byp_E 5co5_A 5co5_B 5co5_D 5co5_G } \\
\text { 5co5_I 5syo_B 5syo_D 2c9t_A 2c9t_B 2c9t_C 2c9t_D 2c9t_E 2c9t_F 2c9t_G } \\
\text { 2c9t_H 2c9t_I 2c9t_J 3peo_B 3peo_G 5bw2_E 5kzu_B 5kzu_E 5jme_A 5jme_B }\end{array}$ \\
\hline
\end{tabular}




\begin{tabular}{|c|c|}
\hline & $\begin{array}{l}\text { 5jme_C 5jme_D 5jme_E 5oaj_A 4ez1_A 4ez1_B 4ez1_C 4ez1_D 4ez1_E 2br8_A } \\
\text { 2br8_B }\end{array}$ \\
\hline 1fqc_A & 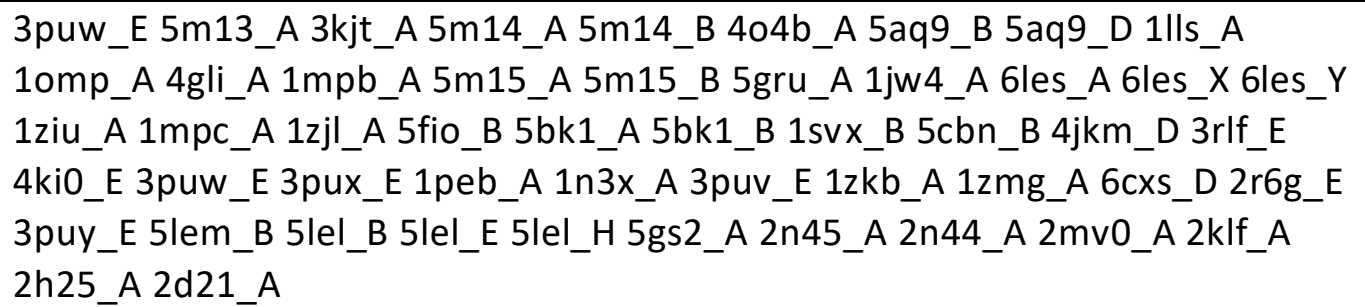 \\
\hline 1ank_B & 4ake_B 4ake_A 4ake_B 4x8h_A 4x8m_A \\
\hline 4hat_C & $\begin{array}{l}\text { 4hb2_C 3m1i_C 5uwp_C 5dif_C 6cit_C 3wyg_C 3vyc_A 5uwi_C 5yst_C 5uww_C } \\
\text { 3wyf_C 3wyf_F 5uwj_C 5di9_C 5uwu_C 5xoj_C 5uwh_C 5dhf_C 5uwr_C 5uwq_C } \\
\text { 5ytb_C 5uwt_C5uwo_C 5uws_C 6a3a_C 5ysu_C 5yro_C 5dh9_C 6a3c_C 6a3b_C } \\
\text { 6a3e_C 5dha_C6a38_C 4hb3_C }\end{array}$ \\
\hline 1g67_B & 1g4e_B 1g4e_B \\
\hline 3hzt_A & 3dxn_A \\
\hline 2brl_A & 3cj0_A \\
\hline 3hqp_P & 1pkl_B 1pkl_B 1pkl_B \\
\hline
\end{tabular}

\section{M-Pro Background}

M-pro cleaves the peptide bond after glutamine residues most frequently occurring in the recognition sequence Leu-Gln-(Ser, Ala, Gly) $)^{34}$. Peptide cleavage by M-pro occurs by a catalytic dyad of cysteine and histidine residues in the active site ${ }^{5}$. While cleavage after glutamine residues has been observed for the main proteases of other coronaviruses, including SARS-CoV ${ }^{6}$ and $\mathrm{MERS}^{7}$, it has not been frequently observed in human proteases ${ }^{4}$. From a therapeutic perspective, this reduces the probability of off-target effects and makes M-pro a promising target for drug design. Most notably, the binding of peptidomimetic inhibitors to the proteolytic active site has been explored as a means to inactivate the protease $^{8}$. By mimicking the geometry of the substrate, these inhibitors become "trapped" in the active site, often by the creation of a covalent bond with the catalytic cysteine ${ }^{8}$, thus 
inactivating the protein. M-pro is an obligate homodimer stabilized by interactions between the N-terminal "finger" residues of one monomer and a glutamate residue in the active site of the opposite monomer ${ }^{39}$. The requirement of dimerization for proteolytic activity presents another opportunity for drug development ${ }^{10}$; the dimer interface has been more recently explored as a potential allosteric drug-binding site ${ }^{11}$.

\section{MTase Background}

The active form of the MTase exists as a heterodimer of the catalytic nsp16 and the activating zinc finger protein nsp $10^{1213}$. Currently, two druggable sites, universal to all protein and nucleotide methyltransferases, have been identified: the SAM cofactor and the RNA cap substrates ${ }^{1214}$. Indeed, several small-molecule inhibitors have been designed to exploit these binding sites with high efficacy in other protein and nucleotide methyltransferases ${ }^{1516}$. Although little functional information exists for the SARS-CoV-2 MTase specifically, it shares a striking $94.5 \%$ sequence identity with the SARS-CoV-1 MTase $^{17}$, which has been functionally well-characterized ${ }^{18}$. Therefore, another potential druggable site-specific on the SARS-CoV-2 MTase is the dimer interface of nsp10 and nsp16, as SARS-CoV-1 MTase activity can be fully diminished by subtle alteration in the interfacial contacts of nsp10 and nsp16 ${ }^{18}$. Although the SARS-CoV-2 MTase crystal structure shows that the interface consists of a large network of polar and hydrophobic contacts, this interface may potentially harbor potent cryptic pockets that can be revealed through MD simulations. 


\section{ArCP Background}

Aryl carrier protein $(\mathrm{ArCP})$ is the first carrier protein domain found in the multidomain protein HMWP2, which is part of yersiniabactin synthetase, the enzymatic system producing yersiniabactin in Yersinia pestis ${ }^{1920}$. Yersiniabactin synthetase is an example of a nonribosomal peptide synthetase (NRPS), which are enzymatic systems in bacteria and fungi that produce a vast number of secondary metabolites. NRPSs are composed of multiple modules organized in assembly-line fashion, such that each module is responsible for adding a single amino or aryl acid onto a forming peptide. A basic module typically contains a carrier protein (CP), an adenylation domain, and a condensation domain ${ }^{19}$. CPs covalently tether a substrate (either the growing peptide, or the molecule being incorporated onto the peptide) via a phosphopantetheine (PP) moiety ${ }^{19}$.

The ArCP tethers a salicylate moiety, and, being the first CP domain, can only exist in one of 3 states: apo, holo, or salicylate loaded. Apo-ArCP is inactive and must be activated via the attachment of PP to $\mathrm{S}^{52}$ by a phosphopantetheinyl transferase ${ }^{19}$. Holo-ArCP refers to ArCP with the PP arm attached. The PP provides a thiol group by which salicylate can be tethered to the $\operatorname{ArCP}$ via a thioester bond ${ }^{19}$. Loaded-ArCP refers to ArCP with salicylate attached to the PP arm. Salicylate is tethered onto holo-ArCP (catalyzed by the stand-alone adenylation domain $\mathrm{YbtE}$ ), generating loaded-ArCP${ }^{19}$. The cyclization domain $\mathrm{Cy} 1$ then catalyzes peptide bond formation between salicylate on ArCP and cysteine on the next CP domain PCP1 19 as well as subsequent cyclodehydration. In the process, salicylate is release from ArCP, regenerating holo-ArCP, and PCP1 harbors hydroxyphenylthiazoline at the end of the reaction. These steps do not occur through a rigid domain architecture; instead, during synthesis ArCP visits its partner catalytic domains through a series of transient 
interactions. Thus, finding small molecule binding sites on $\mathrm{ArCP}$ could disrupt synthesis by disrupting domain communication.

All three forms of ArCP demonstrate a four-helix bundle, with $\alpha 1, \alpha 2$, and $\alpha 4$ lying parallel to one another ${ }^{20} . \alpha 3$ is shorter than the other helices and lies at the significant angle ${ }^{20}$. Loop 1 connects $\alpha 1$ and $\alpha 2$. The PP binding site (Ser ${ }^{52}$ ) is located at the $\mathrm{N}$-terminal end of $\alpha 2^{19}$.

\section{References:}

(1) Cimermancic, P.; Weinkam, P.; Rettenmaier, T. J.; Bichmann, L.; Keedy, D. A.; Woldeyes, R. A.; Schneidman-Duhovny, D.; Demerdash, O. N.; Mitchell, J. C.; Wells, J. A.; Fraser, J. S.; Sali, A. CryptoSite: Expanding the Druggable Proteome by Characterization and Prediction of Cryptic Binding Sites. Journal of Molecular Biology 2016, 428, 709-719. https://doi.org/10.1016/j.jmb.2016.01.029.

(2) Beglov, D.; Hall, D. R.; Wakefield, A. E.; Luo, L.; Allen, K. N.; Kozakov, D.; Whitty, A.; Vajda, S. Exploring the Structural Origins of Cryptic Sites on Proteins. Proceedings of the National Academy of Sciences 2018, 115, E3416-E3425. https://doi.org/10.1073/pnas.1711490115.

(3) Zhang, L.; Lin, D.; Sun, X.; Curth, U.; Drosten, C.; Sauerhering, L.; Becker, S.; Rox, K.; Hilgenfeld, R. Crystal Structure of SARS-CoV-2 Main Protease Provides a Basis for Design of Improved $\alpha$-Ketoamide Inhibitors. Science 2020, 368, 409. https://doi.org/10.1126/science.abb3405.

(4) Rut, W.; Groborz, K.; Zhang, L.; Sun, X.; Zmudzinski, M.; Pawlik, B.; Wang, X.; Jochmans, D.; Neyts, J.; Młynarski, W.; Hilgenfeld, R.; Drag, M. SARS-CoV-2 Mpro Inhibitors and ActivityBased Probes for Patient-Sample Imaging. Nature Chemical Biology 2021, 17, 222-228. https://doi.org/10.1038/s41589-020-00689-z.

(5) Goyal, B.; Goyal, D. Targeting the Dimerization of the Main Protease of Coronaviruses: A Potential Broad-Spectrum Therapeutic Strategy. ACS Comb. Sci. 2020, 22, 297-305. https://doi.org/10.1021/acscombsci.0c00058.

(6) Fan, K.; Ma, L.; Han, X.; Liang, H.; Wei, P.; Liu, Y.; Lai, L. The Substrate Specificity of SARS Coronavirus 3C-like Proteinase. Biochemical and Biophysical Research Communications 2005, 329, 934-940. https://doi.org/10.1016/j.bbrc.2005.02.061.

(7) Wu, A.; Wang, Y.; Zeng, C.; Huang, X.; Xu, S.; Su, C.; Wang, M.; Chen, Y.; Guo, D. Prediction and Biochemical Analysis of Putative Cleavage Sites of the $3 \mathrm{C}$-like Protease of Middle East Respiratory Syndrome Coronavirus. Virus Research 2015, 208, 56-65. https://doi.org/10.1016/j.virusres.2015.05.018.

(8) Liu, Y.; Liang, C.; Xin, L.; Ren, X.; Tian, L.; Ju, X.; Li, H.; Wang, Y.; Zhao, Q.; Liu, H.; Cao, W.; Xie, X.; Zhang, D.; Wang, Y.; Jian, Y. The Development of Coronavirus 3C-Like Protease 
(3CLpro) Inhibitors from 2010 to 2020. European Journal of Medicinal Chemistry 2020, 206, 112711. https://doi.org/10.1016/j.ejmech.2020.112711.

(9) Chen, H.; Wei, P.; Huang, C.; Tan, L.; Liu, Y.; Lai, L. Only One Protomer Is Active in the Dimer of SARS 3C-like Proteinase *. Journal of Biological Chemistry 2006, 281, 1389413898. https://doi.org/10.1074/jbc.M510745200.

(10) Wei, P.; Fan, K.; Chen, H.; Ma, L.; Huang, C.; Tan, L.; Xi, D.; Li, C.; Liu, Y.; Cao, A.; Lai, L. The $\mathrm{N}$-Terminal Octapeptide Acts as a Dimerization Inhibitor of SARS Coronavirus 3C-like Proteinase. Biochemical and Biophysical Research Communications 2006, 339, 865-872. https://doi.org/10.1016/j.bbrc.2005.11.102.

(11) El-Baba, T. J.; Lutomski, C. A.; Kantsadi, A. L.; Malla, T. R.; John, T.; Mikhailov, V.; Bolla, J. R.; Schofield, C. J.; Zitzmann, N.; Vakonakis, I.; Robinson, C. V. Allosteric Inhibition of the SARS-CoV-2 Main Protease: Insights from Mass Spectrometry Based Assays. Angewandte Chemie International Edition 2020, n/a. https://doi.org/10.1002/anie.202010316.

(12) Viswanathan, T.; Arya, S.; Chan, S.-H.; Qi, S.; Dai, N.; Misra, A.; Park, J.-G.; Oladunni, F.; Kovalskyy, D.; Hromas, R. A.; Martinez-Sobrido, L.; Gupta, Y. K. Structural Basis of RNA Cap Modification by SARS-CoV-2. Nature Communications 2020, 11, 3718. https://doi.org/10.1038/s41467-020-17496-8.

(13) Krafcikova, P.; Silhan, J.; Nencka, R.; Boura, E. Structural Analysis of the SARS-CoV-2 Methyltransferase Complex Involved in RNA Cap Creation Bound to Sinefungin. Nature Communications 2020, 11, 3717. https://doi.org/10.1038/s41467-020-17495-9.

(14) Arrowsmith, C. H.; Bountra, C.; Fish, P. V.; Lee, K.; Schapira, M. Epigenetic Protein Families: A New Frontier for Drug Discovery. Nature Reviews Drug Discovery 2012, 11, 384-400. https://doi.org/10.1038/nrd3674.

(15) Zhang, J.; Zheng, Y. G. SAM/SAH Analogs as Versatile Tools for SAM-Dependent Methyltransferases. ACS Chem. Biol. 2016, 11, 583-597. https://doi.org/10.1021/acschembio.5b00812.

(16) Scheer, S.; Ackloo, S.; Medina, T. S.; Schapira, M.; Li, F.; Ward, J. A.; Lewis, A. M.; Northrop, J. P.; Richardson, P. L.; Kaniskan, H. Ü.; Shen, Y.; Liu, J.; Smil, D.; McLeod, D.; ZepedaVelazquez, C. A.; Luo, M.; Jin, J.; Barsyte-Lovejoy, D.; Huber, K. V. M.; De Carvalho, D. D.; Vedadi, M.; Zaph, C.; Brown, P. J.; Arrowsmith, C. H. A Chemical Biology Toolbox to Study Protein Methyltransferases and Epigenetic Signaling. Nature Communications 2019, 10, 19. https://doi.org/10.1038/s41467-018-07905-4.

(17) Mousavizadeh, L.; Ghasemi, S. Genotype and Phenotype of COVID-19: Their Roles in Pathogenesis. Journal of Microbiology, Immunology and Infection 2020. https://doi.org/10.1016/j.jmii.2020.03.022.

(18) Decroly, E.; Debarnot, C.; Ferron, F.; Bouvet, M.; Coutard, B.; Imbert, I.; Gluais, L.; Papageorgiou, N.; Sharff, A.; Bricogne, G.; Ortiz-Lombardia, M.; Lescar, J.; Canard, B. Crystal Structure and Functional Analysis of the SARS-Coronavirus RNA Cap 2'-OMethyltransferase Nsp10/Nsp16 Complex. PLOS Pathogens 2011, 7, 1-14. https://doi.org/10.1371/journal.ppat.1002059.

(19) Goodrich, A. C.; Harden, B. J.; Frueh, D. P. Solution Structure of a Nonribosomal Peptide Synthetase Carrier Protein Loaded with Its Substrate Reveals Transient, Well-Defined Contacts. J. Am. Chem. Soc. 2015, 137, 12100-12109. https://doi.org/10.1021/jacs.5b07772. 
(20) Goodrich, A. C.; Meyers, D. J.; Frueh, D. P. Molecular Impact of Covalent Modifications on Nonribosomal Peptide Synthetase Carrier Protein Communication. Journal of Biological Chemistry 2017, 292, 10002-10013. https://doi.org/10.1074/jbc.M116.766220. 